\title{
ANNUAL SCIENTIFIC CONGRESS 2016
}

$\&$

\section{OBSTETRIC ANAESTHESIA SATELIITE MEETING}

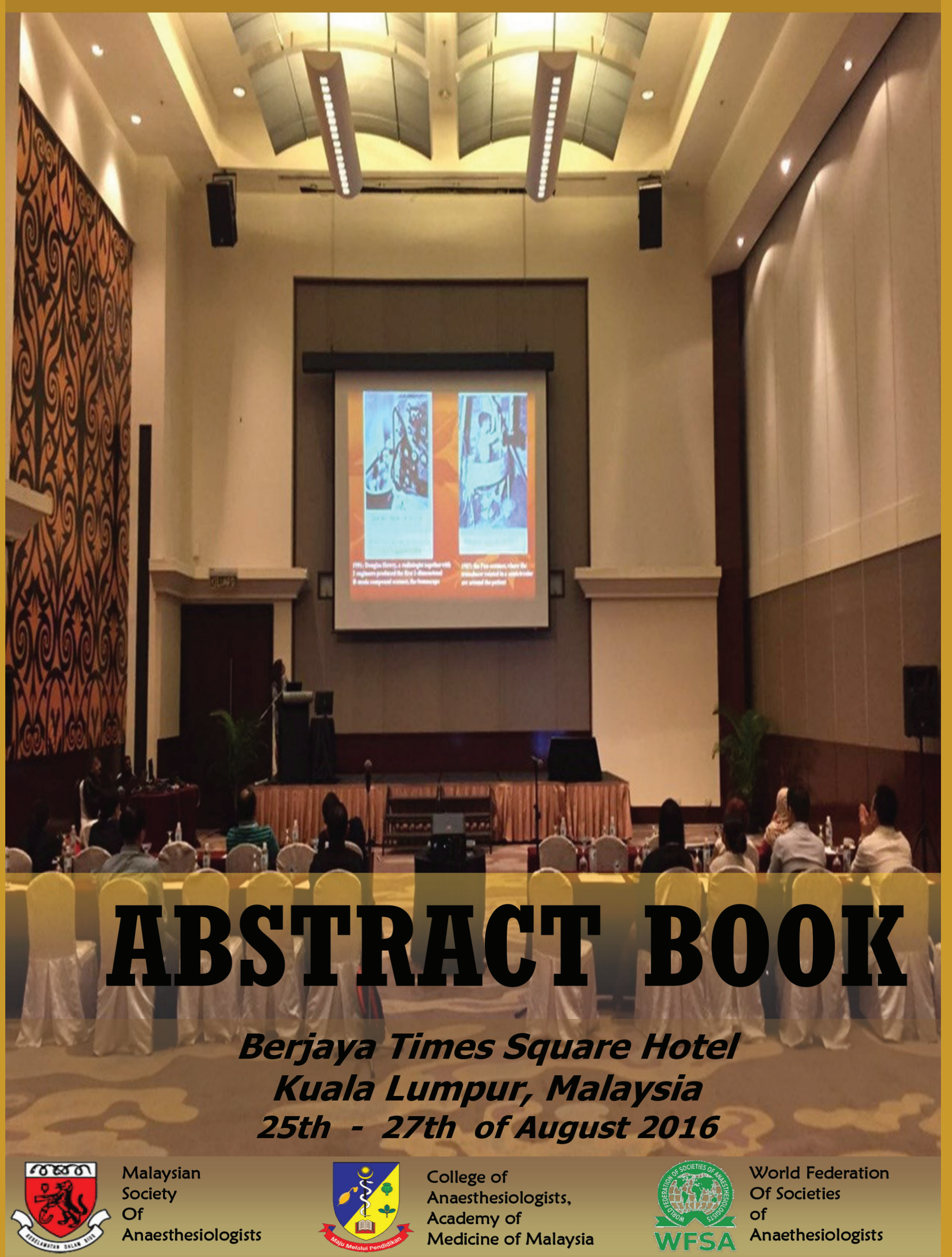




\section{Cover Art for the Annual Scientific Congress and Obstetric Anaesthesia Satellite Meeting 2016}

By Dr Loh Pui San and Cik Nadia Husni Tan, Department of Anaesthesiology, Faculty of Medicine, University of Malaya, 50603 Kuala Lumpur, Malaysia.

Email: lohps@ummc.edu.my

\section{Description:}

The cover picture depicts one of the main lecture halls, Manhattan II in Berjaya Times Square Hotel during a Pre-Congress workshop in the Annual Scientific Congress 2016 and Obstetric Satellite meeting organized jointly by Malaysian Society of Anaesthesiologists, College of Anaesthesiologists and World Federation Society of Anaesthesiologists. 


\section{Foreword}

On the $25^{\text {th }}$ to $27^{\text {th }}$ of August 2016, our Annual Scientific Congress of Malaysian Society of Anaesthesiologists and College of Anaesthesiologists was held successfully at Berjaya Times Square Hotel, Kuala Lumpur with many delegates from all over the country and abroad. The Obstetric Anaesthesia Satellite Meeting, a prestigious international event preceding the $16^{\text {th }}$ World Congress of Anaesthesiologists in Hong Kong was also held in conjunction with our Annual Congress.

Our objective was reflected by the theme entitled 'Back to Basics' to enable a relook at fundamental knowledge in anaesthesia besides keeping up with advances and progress in our field. A panel of international and local speakers who were experts in their respective specialties conducted workshops, lectures, case based discussions and plenaries centred on perioperative care to a packed three-day meeting. This year, we had many non-anaesthetic multidisciplinary topics apart from the usual specialties with speakers from radiology, cardiology, surgery, nephrology and nursing to share their views and improve future collaborative efforts in our clinical work. Research ideas were presented and discussed in the poster and oral free paper sessions to compete for the coveted Best Poster, MSA Award and MSA-AZ Young Investigator Award. Their submitted abstracts now form this special edition with our deepest gratitude to the editors of JUMMEC for making this possible.

Dr Loh Pui San

Chair of Organizing Committee 


\section{JOURNAL OF HEALTH AND TRANSLATION MEDICINE}

(formerly known as Journal of the University of Malaya Medical Centre) JUMMEC

\begin{tabular}{lr}
\hline Special Issue & 2016 \\
\hline Contents & Page \\
Foreword & ii
\end{tabular}

Abstract ID 2

Comparison of King Vision (Channeled Blade) and Glidescope For Tracheal Intubation In Patients

Abstract ID 7

Multiple Recrudescences of Malignant Hyperthermia Crisis: A Successful Management After Incremented Dose of Dantrolene Infusion

\section{Abstract ID 9}

A Leap towards Pain-free District Hospital: Case Series in Paediatric Axillary Block

Abstract ID 12

Comparison Of The Venner A.P. Advance And C-Mac Video Laryngoscopes In Patients Undergoing Tracheal Intubation With Cervical Spine Immobilization

Abstract ID 16

Refeeding Syndrome in A Malaysian Intensive Care Unit: An Assessment of Incidence, Risk Factors and Outcome

\section{Abstract ID 18}

Serial Measurement Of Plasma Neutrophil Gelatinase Associated Lipocalin In Mortality Prediction In Critically III Patients With Systemic Inflammatory Disease And Sepsis

Abstract ID 25

Cranioplasty: It Is More Than Just A Cosmetic Repair

Abstract ID 26

Intraoperative I.V. Paracetamol Reduces Pain Scores, Morphine Consumption And Opioid-Related Side Effects After Supratentorial Craniotomy.

Abstract ID 27

9

Low Volume Interscalene Block: Is It Safe, with Fewer Complications?

Abstract ID 31

An Institutional Survey On The Awareness Of Anaesthetic Doctors On The Specific Considerations In The Management Of Cardiac Arrest During Neurosurgery

Abstract ID 32

Case Reports: The Role Of Costoclavicular Approach Ultrasound Guided Infraclavicular Brachial Plexus Block For Vascular Access Surgery In End Stage Renal Failure Patients With Difficult Airway And Central Venous Disease

A Retrospective Audit Of More Than 1800 Peripheral Nerve Blocks For Neurological Complications In The Largest Tertiary Center In Malaysia 
Complications After Neuraxial Block: A Five-Year Experience In The Largest Tertiary Center In Malaysia

Abstract ID 36

Case Report: Red Dengue

Abstract ID 37

Case Reports: The Role Of Peripheral Nerve Blocks For High Risk Patients Undergoing Surgeries

Abstract ID 38

24 Hour Observation Of Patients After Intrathecal Morphine For Lower Segment Caesarean Section - Is It Overrated?

Abstract ID 39

Maternal Cardiac Arrest And Perimortem Caesarean Delivery: Experience In Terengganu

Abstract ID 41

8

A Study On Evaluating The Cough Evoked Response On Emergence In Patients Undergoing General

Anaesthesia

Abstract ID 42

Report: Two Cases of Malignant Hyperthermia in Hospital Tengku Ampuan Rahimah (HTAR)

Abstract ID 47

Anaesthesia For Lower Segment Caeserean Section In a Patient with Anterior Mediastinal Mass With

Superior Vena Cava Obstruction

Abstract ID 48

Dexmedetomedine For Sedation In Claustrophobic Adults Undergoing Magnetic Resonance Imaging (MRI): A Case Series

Abstract ID 49

Case Report: Jehovah's Witness with von Willebrand Factor Deficiency for Urological Surgery

Abstract ID 50

Case Report: Glycogen Storage Disease Type 1a in Urology Surgery

Use Of Cook Airway Exchange Catheter In Unanticipated Difficult Intubation: Case Report

Abstract ID 53

Peri-operative Anaesthetic Management Of Brain Tumor with Increased Intracranial Pressure and underlying Left Vocal Cord Palsy In Pregnant Lady for Caesarean Section

Abstract ID 54

Randomized Controlled Trial of Ultrasound-Guided Pectoral Nerves Block in Unilateral Breast Surgery with Axillary Clearance - An Interim Report

Abstract ID 57

Impact of Preoperative Anxiety on Propofol Induced Hypotension in Female Patients of Elective Surgery Under General Anaesthesia: Interim Analysis From A Prospective Observational Study

\section{Abstract ID 58}

Case Report on the Retention of A Guidewire: A Rare but Avoidable Complication in Central Venous

Catheterization and our Approach in Management of this Problem in a District Hospital

Selection of the Double-lumen Tube Size for One-lung Ventilation in Asians 
An Audit Of Epidural Analgesia (EA) Among Patients Undergoing Abdominal Surgery in Hospital Umum Sarawak (HUS)

Abstract ID 61

Assessment Of The Depth Of Endotracheal Tube In Pediatric Using Fluoroscopy Technique: A Pilot Study.

Abstract ID 66

Delivering Safe Anaesthesia For Electroconvulsive Therapy During Pregnancy

Abstract ID 67

Underdosing Of Intraoperative Opioids, A Glaring Shortcoming In Perioperative Pain Management

\section{Abstract ID 68}

Implementing Rapid Emergence And Early Extubation From General Anaesthesia

A Crossover Study Comparing In-Plane and Out-of-Plane Techniques for Simulated Ultrasound-Guided Central Venous Cannulation on Phantom Models by Trainees in Anaesthesiology

Abstract ID 71

Postoperative Recovery Profiles After Ambulatory Anaesthesia Using

Spectral Entropy

Abstract ID 73

A Comparison Of Depth Of Muscle Relaxation To Improve Performance Of Thermal Ablation For Liver Tumours

Abstract ID 74

Awake Craniotomy: Are They Satisfied?

Abstract ID 75

Epidural Analgesia for Labour: Comparison between Pre-puncture Ultrasound Localisation versus

Palpation Technique

Abstract ID 77

Clinical Audit on 'Decision-to-Delivery Interval' in Caesarean Sections at Maternity Unit, Hospital Pulau Pinang.

Abstract ID 78

The ABCs Of Managing A Patient With Marquio Syndrome

\section{Abstract ID 79}

The Spreading Of Labor Analgesic Concerns By A Marketing Theory In A Private Japanese Hospital

Abstract ID 80

Turner's With Diabetes Inspidus: A Case Of Severe Underweight With High Water Loss

\section{Abstract ID 81}

Total Intravenous Anaesthesia For Caesarean Delivery: Experience In Hospital Sultanah Nur Zahirah, Kuala Terengganu

Case Report: Successful Resuscitative Hysterotomy in A Parturient with Cardiopulmonary Arrest in the Antenatal Patient Admission Center (PAC) 


\title{
Abstract
}

\section{Abstract ID 2 \\ Comparison of King Vision (Channeled Blade) and Glidescope For Tracheal Intubation In Patients}

\author{
SC Liu', Marzida Mansor ${ }^{2}$ \\ ${ }^{1}$ University of Malaya, Kuala Lumpur, Malaysia \\ 2 University of Malaya, Kuala Lumpur, Malaysia
}

Introduction: Videolaryngoscopes are useful as primary tool for airway management or rescue tool for difficult airway. In this study, we compare two videolaryngoscopes, which are King Vision (channeled blade) and Glidescope. The aim of this study is to compare the intubation performance of King Vision (channeled blade) and Glidescope among patients undergoing elective surgery. The performances of the gadgets were assessed by evaluating the duration for successful intubation and the complication that may arise.

Methodology: 150 patients who underwent elective surgery in University Malaya Medical Centre in year 2014-2015 were enrolled for this study. Patients were randomly allocated to be intubated with King Vision (channeled blade) or Glidescope. The time for successful intubation , number of attempts and complications will be recorded.

Results: The mean time for successful intubation in King Vision (Channeled blade) group was $46.16 \pm$ 24.10 seconds, while for Glidescope group was $47.67 \pm 19.55$ seconds. There was no significant difference between both videolaryngoscopes in term of time for successful intubation. Majority of patients $(92.7 \%)$ can be intubated with 1 intubation attempt. Only 11 patients need 2 intubation attempts. All patients can be intubated successfully with videolaryngoscopes. There was no significant difference between both videolaryngoscopes and number of intubation attempts. Glidescope had higher successful intubation rate (94.7\%) after first attempt compare to King Vision (Channeled blade) (90.7\%). 10\% of intubation with complications noted. 14 cases of bleeding noted, with 9 cases in King Vision (Channeled blade) group and 5 cases in Glidescope group. Only 1 case of dental injury happened in Glidescope group. However, this complication was not statistically significant.

Conclusion: There was no difference in duration for successful intubation and complications in both groups. Both groups had 100\% intubation success rate. We concluded that King Vision (Channeled blade) and Glidescope were similar in efficacy for tracheal intubation in patients. 


\title{
Abstract ID 7 \\ Multiple Recrudescences of Malignant Hyperthermia Crisis: A Successful Management After Incremented Dose of Dantrolene Infusion
}

\author{
Reymi Marseela Abdul Jalil', Zawiah Kassim', Kavitha Kandasamy ' \\ ${ }^{1}$ Segamat Hospital, Johor,Malaysia
}

We experienced a case of malignant hyperthermia $(\mathrm{MH})$ with multiple recrudescences despite on recommended infusion dose of dantrolene $0.25 \mathrm{mg} / \mathrm{kg} / \mathrm{hour}$. A six-year-old healthy boy with no family history of MH susceptibility developed unexplained high ETCO2 after 30 minutes of sevoflurane anaesthesia for toiletting and suturing and $\mathrm{K}$-wire insertion over left ring finger under emergency. Prompt MH diagnosis was made and immediate treatment with dantrolene and other supportive measures was initiated intraoperatively followed by continuous infusion in Intensive Care Unit (ICU). Unfortunately, multiple recrudescences occurred despite on recommended dose of dantrolene which leads to trial of incremented dose to $0.35 \mathrm{mg} / \mathrm{kg} /$ hour which is above recommended dose. The child responded very well with resolution of crisis and this dose was maintained for 24 hours before we considered weaning of dantrolene infusion. The child was successfully discharged after one week of ICU stay. A prompt diagnosis, early administration of dantrolene and vigilant ICU management provided a good outcome in this case. Considering patient variability and the disease spectrum, dantrolene dose should be tailored according to clinical response rather than relying on a certain dose or guideline. 


\title{
Abstract ID 9
}

\section{A Leap towards Pain-free District Hospital: Case Series in Paediatric Axillary Block}

\author{
CY Lean', KT Lee', CK Wong', H Kamarul Zaman'
}

${ }^{1}$ Hospital Sarikei, Sarikei,Sarawak, Malaysia

Objective: To study the safety profile of axillary block performed on paediatric patients; at the same time achieve pain-free anaesthesia.

Methodology: Patients aged two to ten year old, with American Society of Anaesthesiologist Physical Status (ASA ps) I-II were recruited into the study. Patient was induced under general anaesthesia prior to axillary block. The procedure was timed from placement of ultrasound probe until the completion of injection of local anaesthetics. Stimuplex A 22 gauge 2 -inch needle was used and total dose of $0.5 \mathrm{ml} / \mathrm{kg} 0.2 \%$ ropivacaine (maximum $10 \mathrm{ml}$ ) was administered. Vital signs were closely monitored intra- and postoperatively. Pain score was assessed, the time from completion of block to rescue analgesia was noted and any immediate and delayed ( 28 days post procedure) complication was documented.

Result: The mean age of patient is 6.7 year old, with average body weight of 24.1 kilogram (kg). Median duration to perform the block was 4 minutes $(\mathrm{m}) 39$ seconds(s). No immediate complication was documented. Hemodynamic status remained stable intraoperatively. Apart from intravenous fentanyl $1 \mathrm{mcg} / \mathrm{kg}$ given during induction of anaesthesia, no other intravenous analgesia was given intraoperatively. All subjects were documented zero on Face, Legs, Activity, Cry, Consolability scale (FLACC scale) at recovery bay. Mean duration of analgesia lasted 5 hours (hr) $43 \mathrm{~m}$. Mean hospitalization from operation to discharge was 2.7days and no delayed complication recorded.

Conclusion: Paediatric axillary block is considered the safest regional block in upper limb. In this case series, we achieved $100 \%$ success rate with zero incidence of inadvertent vascular puncture, postoperative apnea or other complications. Axillary block offers better pain control without increasing incidence of side effects, in contrast to systemic analgesic drugs. 


\title{
Abstract ID 12 \\ Comparison Of The Venner A.P. Advance And C-Mac Video Laryngoscopes In Patients Undergoing Tracheal Intubation With Cervical Spine Immobilization
}

\author{
JL Khaw', II Shariffuddin', NH Hashim' \\ ${ }^{1}$ University of Malaya, Kuala Lumpur, Malaysia
}

Background: With the increasing availability of video laryngoscopes and improved glottic views, video laryngoscopes were incorporated in the difficult airway algorithms by the American Society of Anesthesiologists in 2013 and Difficult Airway Society in 2015. Numerous video laryngoscopes are available to facilitate tracheal intubation in difficult airways. We compare the intubating characteristics of the Venner A.P. Advance and C-MAC video laryngoscopes in patients undergoing tracheal intubation with difficult airway by simulating cervical spine immobilization.

Methods: We recruited 120 patients into a randomized control trial from June 2015 to December 2015 at the University Malaya Medical Centre, Kuala Lumpur. A trained anesthetic medical officer performed the laryngoscopy by using either APA or C-MAC video laryngoscopes. The primary endpoint was time to successful intubation. Other endpoints were the numbers of intubation attempts, the best glottic view, manoeuvres to aid intubation and the complications associated with tracheal intubation. Analysis includes comparison of results between the APA and C-MAC.

Results: We found shorter intubation times with the C-MAC [median time $21.12 \mathrm{~s}$, inter-quartile range (IQR) 17.13, 27.37, $\mathrm{p}<0.001$ ] compared with the APA [median time 28.46 s, IQR 23.25, 38.12, $\mathrm{p}<0.001$ ]; $95 \% \mathrm{Cl}$ [56.94-57.05]. All the patients were successfully intubated with the C-MAC, whereas $3(5 \%)$ patients failed with the APA, $p$ value 0.172 . The two video laryngoscopes had comparable Cormack and Lehane (CL) grades: $88.1 \%$ achieved a CL grade I view with the C-MAC, versus $80.7 \%$ with the APA, p value 0.269 .

Conclusions: Intubation was significantly faster with the C-MAC video laryngoscope than with the APA video laryngoscopes in patients undergoing tracheal intubation with cervical spine immobilization. However, APA video laryngoscope, with its feature of portability makes it handy and more convenient to bring along for use in pre-hospital settings, ambulances, emergency department and intensive care units. 


\title{
Abstract ID 16 \\ Refeeding Syndrome in A Malaysian Intensive Care Unit: An Assessment of Incidence, Risk Factors and Outcome
}

\author{
Azrina Md Ralib' and Mohd Basri Mat Nor ${ }^{1}$ \\ ${ }^{1}$ Department of Anaesthesiology and Intensive Care, Kulliyyah of Medicine, International Islamic University \\ Malaysia, Kuantan, Pahang
}

Objective: Refeeding hypophosphataemia $(\mathrm{RH})$ is characterised by acute electrolyte derangement following the start of nutrition. Complications associated with this syndrome include heart failure, respiratory failure, paraesthesia, seizure and death. We aim to assess its incidence, risk factors, and outcome in our local intensive care unit (ICU).

Methods: This is a preliminary analysis prospective observational study at the ICU of Hospital Tengku Ampuan Afzan Kuantan. The study was registered under the National Medical Research Register (NMRR-14803-19813) and has received ethical approval. Inclusion criteria includes adult admission longer than 48 hours who were started on enteral feeding in the ICU. Chronic renal failure patients and those receiving dialysis were excluded. RH was considered if plasma phosphate was less than $0.65 \mathrm{mmol} / \mathrm{l}$ within 7 days of ICU admission.

Results: A total of 108 patients were recruited into the study. Of this, 51 (47.2\%) had RH. Patients with $\mathrm{RH}$ had higher SOFA score compared to those without $\mathrm{RH}(7.1 \pm 3.0$ vs $5.7 \pm 3.4, \mathrm{p}=0.02)$. There were no differences in the APACHE II score $(16 \pm 6$ vs $15 \pm 6, p=0.30)$, and in the NUTRIC score $(2.9 \pm 1.7$ versus $2.7 \pm 1.7$, $\mathrm{p}=0.63$ ) between patients with and without $\mathrm{RH}$. Patients with $\mathrm{RH}$ had lower albumin concentration compared to those without $\mathrm{RH}$ ( 23 vs $25, \mathrm{p}=0.04$ ). There were lower trend of magnesium, calcium and potassium concentration, however these were not statistically significant. All four patients with hypomagnesaemia (less than $0.5 \mathrm{mmol} / \mathrm{l})$ had $\mathrm{RH}(\mathrm{p}=0.04)$. There were no differences in mortality, length of hospital or ICU stay and duration of mechanical ventilation.

Conclusion: Refeeding hypophosphataemia is common, occurring in almost half of ICU admission. Patients with RH had higher organ failure score, and lower albumin level. There were no differences in the NUTRIC score and in short-term outcomes. Further studies could evaluate the association between RH and longterm outcome 


\title{
Abstract ID 18
}

\section{Serial Measurement Of Plasma Neutrophil Gelatinase Associated Lipocalin In Mortality Prediction In Critically III Patients With Systemic Inflammatory Disease And Sepsis}

\author{
Azrina Md Ralib' and Mohd Basri Mat Nor ${ }^{1}$ \\ ${ }^{1}$ Department of Anaesthesiology and Intensive Care, Kulliyyah of Medicine, International Islamic University \\ Malaysia, Kuantan, Pahang \\ Introduction: Plasma Neutrophil Gelatinase Associated Lipocalin (NGAL) is the most promising acute kidney \\ injury biomarker to date. NGAL expression is also increased by inflammation and infection. NGAL's ability \\ to predict hard outcome such as mortality in this group of patients is of interest. Sepsis is the leading cause \\ of ICU admission in Malaysia, and contributes to a high mortality rate. Previous studies showed conflicting \\ results on the NGAL's ability to predict of mortality. We hypothesised that serial measurement of biomarker \\ may offer extra advantage compared to a single measurement.
}

Objective: We aim to assess the ability of serial measurement of NGAL in prediction of mortality in critically ill patients with systemic inflammatory diseases.

Methods: This is a secondary analysis of a prospective observational study of Hospital Tengku Ampuan Afzan, Kuantan. The study was registered under the National Medical Research Register (NMR-11-1102-9248) and has received ethical approval. Patients whom fulfill the Systemic Inflammatory Response Syndrome (SIRS) criteria were recruited in the study. Delta NGAL at 24 and 48 hours (DNGAL-24 and DNGAL-48) were defined as 24 and $48 \mathrm{~h}$ NGAL minus Day-1 NGAL.

Results: A total of 151 patients with three days of NGAL measurement were analyzed. Of this, $53(35.1 \%)$ died. Non-survivors were older ( 51 vs $45, p=0.03$ ) and had higher SOFA ( $9 \pm 7$ vs $7 \pm 4, p=0.02$ ) and SAPSII $(47 \pm 15$ vs $40 \pm 15, p=0.01)$ scores compared to survivors. NGAL concentrations over three days were higher in non-survivors compared to survivors (Repeated Measures ANOVA, $\mathrm{p}=0.02$ ). Day 1-NGAL and DNGAL-24 were not independently predictive of mortality. However, DNGAL-48 was predictive after adjusted for age and severity of illness (OR 9.1 (1.97 to $41.7, \mathrm{p}=0.005$ ).

Conclusions: NGAL dynamics over 48 hours independently predicted mortality in critically ill patients with SIRS. This could assist clinicians in risk stratification of this group of high-risk patients. 


\title{
Abstract ID 25
}

\section{Cranioplasty: It Is More Than Just A Cosmetic Repair}

\author{
B Voon ${ }^{1}, \mathrm{PN} \mathrm{Ng}^{2}, \mathrm{P} \operatorname{Tan}^{1}$ \\ ${ }^{1}$ Hospital Umum Sarawak, Kuching, Malaysia \\ 2 Hospital Kuala Lumpur, Kuala Lumpur, Malaysia
}

Background: Cranioplasty after decompressive craniectomy (DC) does not only restore cosmesis and protective function of the skull, it also facilitates rehabilitation of those patients with syndrome of the trephined. However, cranioplasty is associated with a relatively high overall complication rate $(15-36.5 \%)$ and the rate of post-cranioplasty seizures is quoted as $14 \%$.

Case report: A 21-year-old man underwent an elective alloplastic (titanium) cranioplasty following DC performed two years ago for traumatic left frontoparietal subdural haemorrhage. His Glasgow Coma Scale (GCS) score was full. He had mild right hemiparesis but independent in activities of daily living. The awakening after anaesthesia was delayed following the 2.5-hour operation and complicated with tonicclonic seizures, which were aborted with propofol and midazolam. Computed tomographic scan of the brain was done on operating table showed an expanded brain with open cisterns, a mild extradural collection without significant mass effect. The collection of subgaleal drain was $800 \mathrm{ml}$ in the 12 hours postoperative period. He had multiple fitting episodes, which were eventually controlled with dual antiepileptic drugs. Unfortunately, his GCS remained poor necessitated tracheostomy and prolonged rehabilitation.

Discussion: Cranioplasty is associated with changes in cerebrospinal fluid hydrodynamics, cerebral blood flow and cerebral metabolism. Fatal massive cerebral oedema has been reported sporadically following an otherwise uneventful procedure. A negative pressure difference from the elimination of atmospheric pressure that had been chronically applied on the injured sinking brain in combination with the negative pressure created by a subgaleal vacuum drain may cause shifting of the depressed brain to the reconstructed cranial vault and initiate a fatal vasomotor reaction. We postulate that post-cranioplasty seizures illustrated here was attributed to intracranial hypotension explainable by the same mechanisms. Both surgeon and anaesthesiologist should be vigilant and aware of this clinical syndrome. Avoiding the application of suction via the subgaleal drain until the patient is fully awake in recovery bay could potentially minimize this uncommon complication. 


\title{
Abstract ID 26 \\ Intraoperative I.V. Paracetamol Reduces Pain Scores, Morphine Consumption And Opioid-Related Side Effects After Supratentorial Craniotomy.
}

\author{
Mohd Fahmi Lukman', Asmarawati Mohammad Yatim², Mohamed Saufi Awang ${ }^{3}$, \\ Chan Kin Hup ${ }^{3}$, Azura Sharena Yahaya ${ }^{4}$. \\ 'Unit of Anaesthesiology and Critical Care, Faculty of Medicine and Defence Health, National Defence \\ University of Malaysia, Kuala Lumpur, Malaysia. \\ ${ }^{2}$ Department of Anaesthesiology and Intensive Care, HTAA, Kuantan, Pahang, Malaysia. \\ ${ }^{3}$ Neurosurgery Unit, Department of Surgery, Kulliyyah of Medicine, IIUM, Malaysia. \\ ${ }^{4}$ Unit of Radiology, Faculty of Medicine and Defence Health, National Defence University of Malaysia, \\ Kuala Lumpur, Malaysia.
}

Background and objectives: Pain management in craniotomy patients is delicate as these patients are required to be pain free postoperatively but awake for neurological assessment. In contrast to opioids, intravenous Paracetamol (i.v PCM) does not produce sedation and respiratory depression. We hypothesized that intraoperative i.v. PCM might influence postoperative pain scores, morphine consumption and opioidrelated side effects.

Methods: 50 adult patients undergoing elective supratentorial craniotomy were enrolled into the study. Patients were randomized into two groups: i.v. PCM Group ( $n=25)$, and Control Group ( $n=25)$. All patients had general anaesthesia with TCI Propofol - Remifentanil. PCM Group received i.v. PCM 1 gram 30 minutes before skin incision and during skin closure. All patients received IV Fentanyl $1.0 \mathrm{mcg} / \mathrm{kg} 20$ minutes before end of surgery and followed by patient-controlled Morphine. Postoperatively, all patients were admitted into ICU and pain was assessed in the fully awake after extubation (hour 0) and at hour 1,2,4,6,12 and 24 using visual analog score. Morphine consumption and opioids related side effects were recorded within 24 hours after surgery.

Results: 18 patients were excluded as they remained intubated 24 hours after surgery, 32 patients who were extubated immediately included in the analysis. 14 patients (44\%) received intraoperative i.v. PCM, and postoperatively their pain scores at multiple time points were significantly lower $\left(x^{2}(6, N=32)=167.0\right.$, $P<0.001)$ and they had lesser morphine consumption $(Z=-3.001, P=0.03)$ as well. They had lower PONV scores $\left(x^{2}(6, N=32)=33.7, P<0.001\right)$ and less sedated $\left(x^{2}(6, N=32)=158.3, P<0.001\right)$. Their heart rate were significantly lower $(P<0.001)$ but higher systolic blood pressures $(P=0.005)$.

Conclusions: Intraoperative use of i.v. PCM is a useful method of decreasing immediate postoperative pain scores, morphine consumption and opioid-related side effects after supratentorial craniotomy. 


\title{
Abstract ID 27
}

\section{Low Volume Interscalene Block: Is It Safe, with Fewer Complications?}

\author{
CY Lean', JS Ling', N Sabarudin', PY Thang', NI Rahmat', A Huzaifah' \\ ${ }^{1}$ Hospital Sarikei,Sarikei,Sarawak, Malaysia
}

Objective: To assess the safety profile of using low volume local anaesthetics (10ml of $0.5 \%$ levobupivacaine) for interscalene block under ultrasound guidance; at the same time to achieve adequate surgical anaesthesia.

Introduction: Patients aged 12 to 70 years old, with American Society of Anaesthesiologist Physical Status (ASA) I-II were recruited into the study. The patient was required to perform peak expiratory flow rate, and their pupils' size were checked before the interscalene block. Interscalene block was performed with Stimuplex A 22 gauge 2 -inch needle, under ultrasound guidance using $10 \mathrm{ml}$ of $0.5 \%$ levobupivacaine. The procedure duration was timed; and patient was again required to perform peak expiratory flow rate (PEFR) and pupils' size were rechecked after the block was established, and post operatively. Hemodynamic status was closely monitored throughout. Pain score was assessed and any immediate and delayed complication was documented.

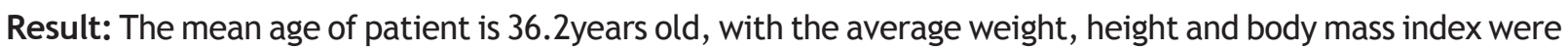
$62.87 \mathrm{~kg}, 1.658 \mathrm{~m}, 22.8718$ respectively. . The mean duration of analgesia was 533 minutes. Hemodynamic status remained stable intra-operatively. The mean baseline PEFR was 337.33litre/minute, upon the establishment of block and at the recovery bay postoperatively were 329.67 litre/minute and 314.67 litre/ minute. The percentage of PEFR reduction post-anaesthesia was $-15.4 \%$ ( $p<0.001)$. All subjects documented 0 pain score despite undergoing surgery using solely regional anaesthesia. No additional analgesics were given intra-operatively. Only $20 \%$ of the subjects showed possible ipsilateral phrenic nerve block $(p<0.001)$. However, there are no adverse respiratory events in the recovery bay.

Conclusion: Interscalene block using low volume local anaesthetics under ultrasound guidance is considered safe in upper limb surgeries and it provides adequate surgical anaesthesia. All these outcomes favour the clinical application of low volume interscalene block in district settings. 


\title{
Abstract ID 31
}

\section{An Institutional Survey On The Awareness Of Anaesthetic Doctors On The Specific Considerations In The Management Of Cardiac Arrest During Neurosurgery}

\author{
K Tan, WNN Shaaya, P Tan \\ Sarawak General Hospital, Kuching, Malaysia
}

Introduction: The incidence of cardiac arrest during neurosurgical procedures is unknown. Though likely to occur in a controlled situation, surgical and positioning factors may pose significant challenges in effective resuscitation.

Objectives and methods: In light of the recently published guidelines for the management of cardiac arrest during neurosurgery in adults by the Resuscitation Council (UK), we surveyed our anaesthetic doctors' awareness of specific considerations involved in this crisis' management. A questionnaire was constructed based on the aforementioned guidelines to be administered to 79 anaesthetic doctors of Sarawak General Hospital, Kuching who have yet to read these recommendations.

Results: The response rate was $78.5 \%(n=62)$, primarily medical officers $(n=53 ; 85.5 \%)$. None of the respondents were aware that the initial dose of adrenaline is to be given in increments (i.e. 50-100 mcg), rather than a $1 \mathrm{mg}$ bolus. This variation from the Advanced Life Support guidelines is meant to limit rebound hypertension and haemorrhage risk should a successful resuscitation be achieved rapidly. Approximately half of the respondents recognised that CPR could be commenced immediately in the prone position while majority (83.9\%) stated that defibrillation but not chest compressions could be effectively performed in the lateral position. The need to change a patient in the sitting to supine position for chest compressions was identified by the most doctors (83.9\%). In a patient whose head is immobilised by a Mayfield ${ }^{\varpi}$ skull clamp, the danger of initiating chest compressions or defibrillation prior to releasing the clamp from the operating table was identified by $64.5 \%$ of the respondents, however only a quarter denied the need to release the patient's head from head pins prior to defibrillation.

Discussion and conclusion: The findings were satisfactory though awareness of our doctors in certain areas could improve. Their learning needs have been addressed via presentation of these guidelines in our department and we wish to extend it to surgeons and theatre staff in near future. We would also like to recommend the inclusion of these scenarios in simulation courses to assist theatre personnel in dealing with these rare yet devastating situations. 


\title{
Abstract ID 32 \\ Case Reports: The Role Of Costoclavicular Approach Ultrasound Guided Infraclavicular Brachial Plexus Block For Vascular Access Surgery In End Stage Renal Failure Patients With Difficult Airway And Central Venous Disease
}

\author{
ZY Beh' \\ ${ }^{1}$ Hospital Kuala Lumpur, Kuala Lumpur, Malaysia
}

Reports: Regional anaesthesia (RA) is the preferred anaesthetic technique for vascular access surgery due to patients often had poor medical health with multiple comorbidities.

Methods: A newly described technique - costoclavicular approach ultrasound guided infraclavicular brachial plexus block was performed in two patients with End Stage Renal Failure (ESRF), who underwent brachio-basilic arteriovenous fistula (BBF) transposition. Both patients had poor comorbidities, American Society of Anaesthesiologists (ASA) physical status III, difficult airway features and central venous occlusive disease. Peripheral nerve blocks were performed with $100 \mathrm{~mm}$ Stimuplex ${ }^{\circledR}$ needle using in-plane approach with ultrasound Sonosite $M$-Turbo ${ }^{\circledR}$ and L38x linear probe. Ropivacaine $0.5 \% 20 \mathrm{ml}$ was delivered at the costoclavicular area. Another $10 \mathrm{ml}$ of Ropivacaine $0.5 \%$ was delivered along the subcutaneous area of proximal medial part of arm near the axilla under ultrasound guidance. Surgical anaesthesia were achieved with the peripheral nerve blocks. Both cases had successfully underwent the surgeries (duration more than 2 hours) with minimal doses of supplemental analgesics, without local anaesthetics infiltration by surgeon at the surgical site. Case 2 required monitored sedation with target controlled infusion Propofol due to long operating time ( 3 hours and 50 minutes).

Conclusions: This technique was a feasible option for regional anaesthesia in vascular access surgery of the upper limb. It might be useful in situation whereby (1) there is difficulty in performing conventional approach (2) RA is perceived to have greater advantages than GA in patients with difficult airway and poor comorbidities. 


\title{
Abstract ID 34
}

\section{A Retrospective Audit Of More Than 1800 Peripheral Nerve Blocks For Neurological Complications In The Largest Tertiary Center In Malaysia}

\author{
ZY Beh', LJ Tan' , AM Azidin', ANM Kamil', S Velayuthapillai' ${ }^{1}$ M Shahnaz Hasan ${ }^{2}$, KU Ling ${ }^{3}$, \\ S Fathil ${ }^{4}$ \\ ${ }^{1}$ Hospital Kuala Lumpur, Kuala Lumpur, Malaysia \\ 2 University of Malaya, Kuala Lumpur, Malaysia \\ ${ }^{3}$ Ramsay Sime Darby Ara Damansara Medical Centre, Selangor, Malaysia \\ ${ }^{4} \mathrm{Ng}$ Teng Fong General Hospital, Jurong East, Singapore
}

Background and Objective: Our institution had seen a surge in number of patients had postoperative neurological symptoms (PONS) after peripheral nerve blocks (PNBs). The hype was many pinpoint as block related nerve injury. Hence we carried out a retrospective audit on all patients received PNBs over the last two years.

Methodology: This was an institutional review with waiver of informed consent. Ethical approval was not required. The audit period were from $1^{\text {st }}$ January 2014 until $31^{\text {st }}$ December 2015 and comprised of two parts: First, description of total number of patients received PNBs, total number of blocks performed and number of patients who developed PONS. Second, review the cohort of patients required follow up for PONS after PNBs in the anaesthetic clinic. The independent expert panel would evaluate their summarized information. Overall findings were presented in quantitative analysis in combination with literature interpretation, case review (clinical vignettes) and expert opinion.

Results: A total of 1435 patients received PNBs and 1,856 blocks were performed on them (highest number in Malaysia). 36 patients developed PONS, giving incidence of 19 per 1000 blocks. 28 of them had persistent numbness beyond the expected duration of block (78\%). The rest had combined motor weakness and numbness. Most of them recovered few days after operation and discharged without the need for clinic follow up.

17 patients required follow up. 7 of them had electrodiagnostic study. Detailed analysis with expert feedback, majority were surgical related nerve injury, 4 patients had significant patient related factor. None had blocked related nerve injury. Most had full recovery and many defaulted subsequent follow up.

Conclusion: PONS was common with most had sensory paraesthesia which resolved within few weeks postoperatively. Most were likely due to non anaesthesia related cause than nerve block related nerve injury. There is definite need to have guideline to manage patients with PONS. 


\title{
Abstract ID 35 \\ Complications After Neuraxial Block: A Five-Year Experience In The Largest Tertiary Center In Malaysia
}

\author{
ZY Beh', LJ Tan', HX Chang', YW Lim', AR Thohiroh', S Velayuthapillai', MN Norliza ${ }^{2}$, M \\ Shahnaz Hasan ${ }^{3}$, S Fathil ${ }^{4}$ \\ ${ }^{1}$ Hospital Kuala Lumpur, Kuala Lumpur, Malaysia \\ 2 Hospital Selayang, Selangor, Malaysia \\ 3 University of Malaya, Kuala Lumpur, Malaysia \\ ${ }^{4} \mathrm{Ng}$ Teng Fong General Hospital, Jurong East, Singapore
}

Background: Although complications after central neuraxial blocks (CNBs) are extremely rare, but when they occur, they often result in life-altering injuries.

Objective: We attempt to identify the incidences of neuraxial complications in the largest institute in Malaysia.

Methodology: This was an institutional review with waiver of informed consent. Ethical approval was not required. The audit period were from $1^{\text {st }}$ January 2011 until 31 $1^{\text {st }}$ December 2015 and comprised of two parts: First, an assessment of the number of CNBs performed throughout five year period (for denominator information) and an audit of the major complications of these procedures performed during same period (for numerator information). Second, review the cohort of obstetric patients who had accidental dural puncture (ADP) and postdural puncture headache (PDPH) following CNBs. The independent expert panel would ascertain causation, severity and outcome of each cases with complication. Overall findings were presented in quantitative analysis in combination with literature interpretation, case review (clinical vignettes) and expert opinion.

Results: A total of 37481 patients received central neuraxial blocks during five-year period. $79.5 \%$ of them spinal, 12.3\% epidural, 3.2\% CSE and 5\% combined GA with epidural or caudal. 75 patients developed postoperative complications after neuraxial blocks. Two unlikely related to neuraxial blocks. 10 had major complications - 1 case of epidural hematoma (giving incidence of 2.7 per 100,000 neuraxial blocks), 9 cases of cardiovascular collapse (whether direct or indirect to CNBs - e.g. high spinal, confounding sepsis; with 1 death on table). The most common minor complications were ADP with PDPH, followed by transient neurological symptoms (TNS), retained epidural catheter and backache. Identifiable risk factors: female, obstetric and extreme body mass index (BMI) patients. 1 case had filed complaint to the hospital authority.

Conclusion: This was the first baseline database for Malaysian population on neuraxial complications. Major limitation was poor data entry and it's a retrospective analysis. There's a great need for proper documentation and data collection system (prospective, digitalized information). 


\title{
Abstract ID 36
}

\section{Case Report: Red Dengue}

\author{
SC Tan, CW K'ng
}

Hospital Seberang Jaya, Pulau Pinang, Malaysia

Introduction: Dengue infection is a mosquito-borne viral infection that endangers an estimated 2.5 billion people worldwide. Clinical manifestations of dengue ranges from a febrile illness to a life-threatening condition characterized by extensive plasma leakage.

Methods: A 37-year-old Malay man presented to us with dengue fever complicated by acute massive upper gastro-intestinal bleeding. Such cases are associated with high mortality. His resuscitation involved transfusion of 25 pints of packed cell, 2 pints of whole blood, 4 units of fresh frozen plasma, and 7 units of platelet in total, over a period of 8 days due to profuse, recurrent upper gastrointestinal bleeding. Early diagnostic endoscopy was performed and medical treatment including the use of intravenous vitamin $\mathrm{K}$, tranexamic acid and desmopressin remained the main management.

Discussion: The purpose of this case report is to highlight the importance of early identification and resuscitation of dengue patients complicated by significant gastrointestinal bleed. Packed cell is preferred over whole blood, however prophylactic blood transfusion is not advised. In such cases, early diagnostic endoscopy is beneficial to determine the severity and site of gastrointestinal bleeding, but therapeutic endoscopy runs the risk of worsening the bleed.

Conclusion: In conclusion, early identification, resuscitation and medical treatment remain the mainstay of management in dengue patient with significant upper gastrointestinal bleed. 


\title{
Abstract ID 37 \\ Case Reports: The Role Of Peripheral Nerve Blocks For High Risk Patients Undergoing Surgeries
}

\author{
ZY Beh' K Anwar ${ }^{2}$ \\ ${ }^{1}$ Hospital Kuala Lumpur, Kuala Lumpur, Malaysia \\ 2 Hospital Sultanah Aminah, Johor Bahru, Malaysia
}

Reports: Peripheral nerve blocks (PNBs) are widely-used for surgical anesthesia as well as for both postoperative analgesia. PNBs offer distinct benefits over general or neuraxial anesthesia in certain clinical situations, especially high risk patients. We described three cases received PNBs for their surgical conditions posted as emergency cases.

Case A: A 60 years-old male with CCF, echocardiography EF 40\%, NYHA Class III, difficult airway features with OSA symptoms - STOP BANG score 5 . He underwent saucerization of a small carbuncle at occipital area of scalp. Monitored sedation with scalp block was performed (bilateral greater occipital nerve and lesser occipital nerve blocks, landmark technique). Operation successful, intraoperative haemodynamics stable with minimal use of supplemental analgesia. Patient's outcome - discharged home

Case B: A 78 years-old lady with end stage renal failure. She presented with left BCF hematoma, quick expanding and resulted her to develop NSTEMI secondary to anaemia. Patient was transfused with blood. No coagulopathy. Ultrasound guided supraclavicular combined with infraclavicular brachial plexus was given to ensure surgical anaesthesia as patient had poor sonoanatomy view. Surgical evacuation was successfully done without supplemental analgesia. Stable haemodynamics. Patient's outcome - stable, came back for second wound debridement under PNBs.

Case C: A 75 years-old man who underwent CABG complicated with biventricular heart failure with pulmonary hypertension (PASP $51 \mathrm{mmHg}$ ), persistent cardiogenic shock on dobutamine and noradrenaline infusion, echocardiography EF 21\%. Patient had infected wound at left saphenous graft area required wound debridement and daily dressing. Ultrasound guided femoral and sciatic nerve block was performed. Catheter infusion was inserted for both nerves running at $1 \mathrm{ml} / \mathrm{hr}$ Ropivacaine $0.2 \%$. Patient's pain score was 0 . Patient eventually died due to cardiac problem.

Conclusions: There is definite role of PNBs for high risk patients undergoing surgeries but careful case selection, block feasibility, alternative back up plan and standard monitoring must be employed all the time. 


\section{Abstract ID 38}

\section{Hour Observation Of Patients After Intrathecal Morphine For Lower Segment Caesarean Section - Is It Overrated?}

Kong Shu Ning', Tee Shi Ting', Muhammad Faiz Bin Ismail', Joanne Sue James', Hamitra Gandhi', Thiruselvi Subramaniam', Jenny Tong May Geok ${ }^{2}$

${ }^{1}$ International Medical University Clinical School, Seremban, Malaysia

${ }^{2}$ Hospital Tuanku Jaafar Seremban, Seremban, Malaysia

Background: Intrathecal morphine improves the management of post-surgical pain. However, side effects of morphine can be dangerous and distressing to patients. 24-hour monitoring by acute pain service (APS) team is conducted for early detection and intervention of life-threatening side effects like respiratory depression. With increased use of intrathecal morphine for lower segment caesarean sections (LSCS) and increased patient: staff ratio, attention to patients on other forms of pain management can get delayed.

Objectives: This study aims to identify the side effects of intrathecal morphine at 0-6 hours, 6-12 hours or 12-24 hours after administration for LSCS and to determine the incidence of respiratory depression. We also aim to compare side effects and pain score among different races.

Method: A cross-sectional study was conducted on 323 patients (18 to 45 years old) who received intrathecal morphine for LSCS in Hospital Tuanku Jaafar, Seremban. An interviewer-centered questionnaire was provided and demographic information was obtained. The presence and nature of side effects were noted. The data was analysed using SPSS statistical software.

Results: $80 \%(\mathrm{n}=259)$ patients experienced the side effects of intrathecal morphine. Pruritus is the most common side effect ( $88 \%$; $n=227)$. Side effects commonly occurred during the first 6 hours, $94 \%(n=244)$. There were no patients with respiratory depression in this study. We also found that there is statistically significant more occurrence of side effect among the Malay $(p=0.004)$.

Conclusion: We found that most side effects caused by intrathecal morphine occurred within 12 hours of placement. We conclude that 12 -hour monitoring by APS team is adequate before discharging from the APS care and regular monitoring can be continued in the ward by the parent team. Further study with a bigger sample size may be needed especially to look at the racial predominance of side effects that was noted in our study. 


\title{
Abstract ID 39
}

\section{Maternal Cardiac Arrest And Perimortem Caesarean Delivery: Experience In Terengganu}

\author{
WAA Wan Md Adnan', N Razak', MH Zakaria', \\ ${ }^{1}$ Department of Anaesthesia \& Intensive Care, Hospital Sultanah Nur Zahirah, K Terengganu, Malaysia.
}

Introduction: Maternal cardiac arrest is a traumatic experience for patient, family and care providers. AHA resuscitation guidelines recommend Perimortem Caesarean Delivery (PCMD) to be promptly performed to improved maternal outcome. There are limited experiences with regards to PCMD in Malaysia, apart from case reports.

Report: A 26-year-old lady with history of admission due to hypertensive emergency. She was planned for emergency Caesarean delivery for fetal distress. Upon arrival to operation theatre, patient was in respiratory distress, due to acute pulmonary oedema with hypertensive crisis. Decision was made for immediate intubation. She went into cardiac arrest after intubation due to hypoxia. High quality cardiopulmonary resuscitation was commenced including left uterine displacement. PMCD was performed after 10 minutes of resuscitation efforts fail. A live baby boy with poor Apgar score was born. Soon after fetal delivery, return of spontaneous circulation (ROSC) was achieved. Patient was ventilated in intensive care unit and extubated after 4 days. CT Imaging of the brain during ICU stay revealed occipital lacunar infarction. She was transferred to High Dependency Unit, with the GCS of 10/15. Patient slowly recovered her full conscious level. She was able to be discharged home with no motor weakness after 25 days from the life threatening event. Her baby was also discharged home few days later.

Conclusion: This is a valuable experience with regards to PMCD in our hospital, and a very rewarding outcome to salvage the life of both mother and son. PMCD gave profound benefit to the survival of the parturient even though the delivery is delayed than the recommended time. 


\title{
Abstract ID 41
}

\section{A Study On Evaluating The Cough Evoked Response On Emergence In Patients Undergoing General Anaesthesia}

\author{
MM Miskan', PS Loh² \\ 1 Hospital Umum Sarawak, Kuching, Malaysia \\ 2 University of Malaya, Kuala Lumpur, Malaysia
}

Objectives: Coughing during emergence from general anaesthesia may cause unfavorable side effects namely; sore throat, nausea, surgical site bleeding and increase in intracranial and intraocular pressure. To compare the incidence and severity of cough on emergence in patients undergoing general anesthesia receiving either neostigmine or sugammadex in a bolus or staggered dose for their reversal. Risk factors for severe cough on emergence are determined as the secondary objective.

Methods: This was a randomized control trial performed in the operation theatre of University Malaya Medical Centre. 120 ASA 1-3 patients, 18-80 years old, undergoing general anaesthesia and planned for extubation were included and randomized to three groups of reversal:

A- sugammadex $1 \mathrm{mg} / \mathrm{kg}$ on emergence and another $1 \mathrm{mg} / \mathrm{kg}$ immediately post extubation

B- sugammadex $2 \mathrm{mg} / \mathrm{kg}$ on emergence

C- neostigmine $2.5 \mathrm{mg} /$ glycopyrrolate $200 \mathrm{mcg}$ as control

Perioperative and demographic data, incidence and severity of cough on emergence, sedation score, vital signs and reversal time were recorded and analyzed. Patients were followed up until discharge to ward. Postoperative cough, sore throat, agitation, vital signs and postoperative nausea and vomiting were monitored in recovery.

Results: Staggered dose of sugammadex compared to bolus dose showed less incidence of severe cough with good safety profile. Sugammadex $2 \mathrm{mg} / \mathrm{kg}$ as a single bolus showed a significantly higher incidence of severe cough on emergence compared to the other 2 groups (OR: $11,95 \% \mathrm{Cl}: 3.8-31.7, \mathrm{P}<0.001$ ). Other risk factors for cough include male, and smokers. No significant difference was seen in vital signs or adverse events on emergence.

Conclusion: Staggered dose of sugammadex is safe and can avoid the hazards of severe cough on emergence. 


\title{
Abstract ID 42 \\ Report: Two Cases of Malignant Hyperthermia in Hospital Tengku Ampuan Rahimah (HTAR)
}

\author{
Dr M F Ibrahim' Dr M R Zainol Abidin', Dr N Sangit ${ }^{1}$ \\ ${ }^{1}$ Hospital Tengku AmpuanRahimah, Klang, Malaysia
}

Report: Malignant hyperthermia $(\mathrm{MH})$ is a rare genetic disorder that widely feared yet rarely encountered. Interestingly, we encountered two cases of malignant hyperthermia during anaesthesia in our centre. Our first case involved a 14 year old Malay boy who underwent multiple procedures following motor vehicle accident in which he sustained fractures over the right humerus and foot. Following administration of suxamethonium, he developed masseter muscle spasm, followed by unexplained tachycardia, hypercarbia and hyperthermia. Our second case involved a 28 year old Chinese male who underwent laparoscopic appendicectomy. Unlike the first case, the onset of symptoms for this patient occurred later during the anaesthesia. In both cases, we monitored their temperatures using nasopharyngeal probe only after suspecting $\mathrm{MH}$. We found that skin temperature monitoring was not accurate. We recommend core temperature monitoring for any case of general anaesthesia lasting more than 30 minutes. A high incidence of mortality was associated with MH but with early recognition and treatment strategies, mortality can be prevented 


\title{
Abstract ID 47
}

\section{Anaesthesia For Lower Segment Caeserean Section In a Patient with Anterior Mediastinal Mass With Superior Vena Cava Obstruction}

\author{
Lin Jun Tan', Thohiroh Abdul Razak' \\ ${ }^{1}$ Department of Anaesthesiology, Hospital Kuala Lumpur, Kuala Lumpur, Malaysia
}

Report: Pregnancy is associated with both anatomical and physiological changes in the body, especially in cardiovascular and respiratory systems. Anterior mediastinal mass is a rare pathology associated with superior vena cava (SVC) obstruction. The combination of both (pregnancy and mediastinal mass) in a single patient presents an unusual anaesthetic challenge. We present a case of term pregnant lady presented with anterior mediastinal mass with signs and symptoms of SVC obstruction complicated with thrombus in SVC. Patient underwent lower segment caesarean section with cardiothoracic surgeon and cardiopulmonary bypass machine standby under combined spinal epidural anaesthesia with a favorable feto-maternal outcome. This paper discuss about the perioperative consideration for a parturient presented with anterior mediastinal mass with SVC obstruction. The report also illustrates the importance of multidisciplinary team involvement to ensure the successful outcome of the pregnancy.

Keywords: anterior mediastinal mass, SVC obstruction, multidisciplinary team, combined spinal epidural 


\title{
Abstract ID 48
}

\section{Dexmedetomedine For Sedation In Claustrophobic Adults Undergoing Magnetic Resonance Imaging (MRI): A Case Series}

\author{
Mohd Azlan Ariffin (MD)', Siti Nadzrah Yunus (MBBS)', Pui-San Loh (FANZCA)', ${ }^{*}$, Vineya \\ Rai (M.med)', Lucy Chan (FANZCA)', Norlisah Ramli (FRCR) ${ }^{2}$ \\ ${ }^{1}$ Department of Anaesthesiology, Faculty of Medicine, University Malaya, 50603 Lembah Pantai, Kuala \\ Lumpur, Malaysia \\ 2 University Malaya Research Imaging Centre, Department of Biomedical Imaging, Faculty of Medicine, \\ University Malaya, 50603 Lembah Pantai, Kuala Lumpur, Malaysia
}

Background: Claustrophobic reactions are a major obstacle to performing magnetic resonance imaging (MRI) examinations. Conscious sedation provides a solution for higher success and safety rates for MRI in this group of patients.

Objective: To determine the efficacy and safety of Dexmedetomedine as sedation for claustrophobic adults undergoing MRI.

Methods: 15 claustrophobic ASA I-III adults with a history of anxiety or panic attacks in enclosed space especially during previous MRI studies were included in this case series. All were electively planned for MRI in University Malaya Medical Centre by their primary teams. A loading dose of $1.0 \mathrm{mcg} / \mathrm{kg}$ over 10 minutes, followed by maintenance of $0.2 \mathrm{mcg} / \mathrm{kg} / \mathrm{hr}$ of Dexmedetomedine was administered to achieve a score of 3 in Ramsay Sedation Score (RSS) following Precedex (Hospira Inc) guidelines for sedation based on their ideal body weight. Anxiety levels were determined with Visual Anxiety Score (VAS) and the Spielberger Strait Test Anxiety Inventory (STAI) taken at 2 time points - 5 minutes after entering the scan room and on arrival to the recovery. Patient Satisfaction Scores were also charted and the images evaluated by an experienced neuroradiologist.

Results: 15 patients were recruited, 14 out of which completed their scans $(93.3 \%)$. One case was rescheduled due to undiagnosed obstructive sleep apnea. Significant reduction of anxiety levels, VAS and STAI was shown (mean difference 5.50 and 33.71 respectively, $p<0.001$ ). Time taken to achieve RSS 3 was 10.71 minutes (SD 4.63) with a mean dose of $0.44 \mathrm{mcg} / \mathrm{kg} / \mathrm{hr} .2$ cases had hypotension (NNH 7) and one developed bradycardia (NNH 14). The average score for image quality was 3.57 (SD 0.76 ) with 2 only satisfactory images. Patient Satisfaction Scores were 12.79 (mean rank).

Conclusions: Dexmedetomedine can effectively reduce anxiety in claustrophobic adults undergoing MRI. Hypotension and bradycardia are commonly observed whilst the image quality may only be satisfactory. 


\title{
Abstract ID 49
}

\section{Case Report: Jehovah's Witness with von Willebrand Factor Deficiency for Urological Surgery}

\author{
Farah $R^{1}$, Radhi $F^{1}$, Suzy $B^{1}$, Normi $S^{1}$, Norzalina $E^{1}$ \\ ${ }^{1}$ Department of Anaesthesiology and Intensive Care, Sarawak General Hospital
}

Introduction: The optimal perioperative anaesthetic management for patient with Jehovah's Witness faith should be acknowledged to various strategies to reduce blood loss and thus blood transfusion. Our respect for patient's autonomy and human rights is fundamental to good medical practice.

Case Description: A 24 years old devout Jehovah's Witness male, required rigid cystoscope and left ureterolithotripsy for left renal stone. He has underlying Glycogen Storage Disease Type 1a (GSD), vonWillebrand factor deficiency Type 1, hepatic adenoma, and osteoporosis. He had history of dental extraction few years ago without any complications. Preoperatively, vital signs were stable, with admitting haemoglobin of $14.7 \mathrm{~g} / \mathrm{dl}$, hematocrits $41.8 \%$, platelet 280000 , and normal prothrombin time. Prior to surgery, infusion desmopressin $0.3 \mathrm{mcg} / \mathrm{kg}(18 \mathrm{mcg})$ was given over 30 minutes. General anaesthesia was administered with arterial blood pressure as invasive monitoring. Surgery lasted for two hours with minimal blood loss. He was discharged home well at postoperative day two.

Discussion: Techniques and alternatives are available for anaesthetist to plan and manage Jehovah's Witness choice of non-blood management. Interdepartmental discussion on how to minimise blood loss with haematologist regarding use of desmopressin as alternative to blood products, and for surgeon to do minimally invasive or staging surgery. Consultant anaesthesiologist was advised regarding the impending surgery. Desmopressin is a synthetic analog of anti diuretic hormone which elevates factor VII and vonWillebrand factor. Most important is the established patient's awareness of risks of refusal towards blood transfusion.

Conclusion: Management of Jehovah's Witness should be planned as early as possible to ascertain the degree of limitation on intraoperative management. A protocol should be inaugurated in a hospital or health care facility. 


\title{
Abstract ID 50
}

\section{Case Report: Glycogen Storage Disease Type 1 a in Urology Surgery}

\author{
Suzy $B^{1}$, Wilson $M^{1}$, Farah $R^{1}$, Normi $S^{1}$, Norzalina $E^{1}$ \\ ${ }^{1}$ Department of Anaesthesiology and Intensive Care, Sarawak General Hospital, Kuching, Sarawak
}

Introduction: Glycogen storage disease Type (GSD-1a) 1a is a rare autosomal recessive inherited disorder with an absent gene expression of glucose-6-phosphate enzyme complex. The liver cannot metabolize stored glycogen leading to poor tolerance to fasting due to increased risk of hypoglycaemia and lactic acidosis. We described the anaesthetic considerations in a GSD-1a patient.

Case Report: Twenty-four years old Jehovah's Witness with underlying Von Williebrand(VWB) disease Type 1, GSD related hepatic adenoma and hyperuricaemia, was scheduled for right cystoscopy and left ureterolithotripsy of bilateral renal stone under general anaesthesia. He was fasted for six hours with intravenous infusion of $5 \%$ dextrose saline at $104 \mathrm{ml}^{\text {.hr-1 }}{ }^{-1}$ but allowed glucose drinks up to two hours before surgery. He was given desmopressin $0.3 \mathrm{mcg} / \mathrm{kg}$ thirty-minute pre-induction for his VWB disease. Surgery lasted for two hours and he remained euglycaemic with intravenous infusion of 5\% dextrose saline at 104 $\mathrm{ml} . \mathrm{hr}^{-1}$ which was continued post-operatively until he tolerated orally. Recovery from anaesthesia was uneventful. Venous blood gas in ward showed mild metabolic acidosis which resolved after bolus one pint of $0.9 \%$ normal saline.

Discussion: In GSD-1a patient, fasting period and surgical stress results in glucose-6-phosphate accumulation in the blood causing hypoglycaemia and lactic acidosis. Our patient remained normoglycaemic by limiting fasting to solid food for six hours but allowing glucose drinks, in addition to intravenous glucose infusion, until two hours prior to surgery. Acidosis was resolved by maintaining adequate hydration using non lactatecontaining fluids.

Conclusion: A multidisciplinary approach by anaesthesiologists, surgeon, endocrinologist, haematologists and nephrologists is the key of success in managing GSD patient. 


\title{
Abstract ID 51
}

\section{Use Of Cook Airway Exchange Catheter In Unanticipated Difficult Intubation: Case Report}

\author{
HS Hoong', SLS Soon', Faizal Hisham', CW Soo', LMH Ng'
}

${ }^{1}$ Hospital Queen Elizabeth, Kota Kinabalu, Malaysia

Background: Unanticipated difficulty in tracheal intubation poses a true challenge to anaesthesiologists. Narrowing or obstruction of the airway just below the vocal cords presents difficulty in airway management, and often requires a cricothyroidotomy or tracheostomy. Many guidelines have been published to provide strategies in managing difficult airways. However little has been mentioned about the usage of the Cook Airway Exchange Catheter (CAEC) as an airway adjunct.

Report: This case report demonstrates the use of a CAEC in an unanticipated emergency situation where even the smallest size microlaryngeal tube failed to advance beyond the vocal cords. We attempted to oxygenate through a CAEC by using high-pressure manual bagging, with capnogram monitoring, while an emergency tracheostomy was performed. 


\title{
Abstract ID 53 \\ Peri-operative Anaesthetic Management Of Brain Tumor with Increased Intracranial Pressure and underlying Left Vocal Cord Palsy In Pregnant Lady for Caesarean Section
}

\author{
Katherinnragon $A^{1}$, Dayang $S^{1}$, Suhaimi $T^{1}$, Haizar $K^{1}$, Norzalina $E^{1}$ \\ ${ }^{1}$ Anaesthesiology Department, Hospital Umum Sarawak, Kuching, Sarawak
}

Introduction: Brain tumour with increased intracranial pressure (ICP) combined with vocal cord palsy will pose a peri-operative challenge especially in managing a patient requiring Caesarean section.

Case presentation: A 27 year old lady, Gravida 2 Para 1 at 38 weeks gestation, presented with generalized tonic clonic seizure which aborted spontaneously after 30 minutes. It was her fourth presentation of seizure since 1 year ago but never sought for any treatment. She also had left vocal cord palsy for investigation. Magnetic Resonance Imaging (MRI) showed large left parietal extra axial mass with adjacent smaller homogenous enhancing lesion associated with marked ipsilateral white matter edema. She was started on Keppra 500miligrams (mg) twice daily and planned for Caesarean Section after multidisciplinary discussion. Preoperatively, she was premedicated with gastric acid prophylaxis and planned for rapid sequence induction. Induction done with intravenous thiopentone $150 \mathrm{mg}$, suxamethonium $50 \mathrm{mg}$ and started on target controlled infusion $(\mathrm{TCl})$ of remifentanil $2 \mathrm{ng} / \mathrm{ml}$ at $7 \mathrm{mls} /$ hour. Anaesthesia was maintained with Sevofluorane 1.0vol\% and TCl remifentanil $2 \mathrm{ng} / \mathrm{ml}$ at $7 \mathrm{mls} /$ hour. Intraoperative was uneventful. She was subsequently admitted to intensive care unit for close monitoring and early extubation. Post-operatively, she was started on patient controlled analgesia-morphine (PCAM). She then underwent excision of brain tumor a week after. Further investigation revealed that she has stage 4B lung adenocarcinoma with brain metastasis and right gonadal vein thrombosis and which she subsequently underwent inferior vena caval filter insertion.

Conclusion: Pregnant mother with a brain tumor should be kept normotensive and pain-free to prevent further increase of ICP. The mode of anaesthesia and agent of induction need to be considered properly especially in patient with high risk of aspiration and raised ICP. Correct choice of thromboprophylaxis also needs to be considered in a patient at risk of thromboembolic event. 


\title{
Abstract ID 54
}

\section{Randomized Controlled Trial of Ultrasound-Guided Pectoral Nerves Block in Unilateral Breast Surgery with Axillary Clearance - An Interim Report}

\author{
Jabbar $\mathrm{Al}^{1}$, Soo $\mathrm{CW}^{1}$, Cheah $\mathrm{SL}^{1}$, Shim $\mathrm{JY}^{1}$, Fong $\mathrm{KK}^{1}$, $\mathrm{LMH} \mathrm{Ng}^{1}$ \\ ${ }^{1}$ Hospital Queen Elizabeth, Kota Kinabalu, Sabah, Malaysia
}

Background: Pectoral nerves block (PECS) might confer better pain control in breast surgery with less systemic side effects compared with standard anaesthetic practice with using IV opioids.

Methods: This prospective, randomized controlled trial, aims to enroll 60 patients with primary inclusion criteria of unilateral breast surgery with axillary clearance randomized into treatment group receiving PECS block with ropivacaine $0.375 \%$ and control group receiving IV morphine $0.1 \mathrm{mg} / \mathrm{kg}$ at the start of surgery.

Objectives: To compare the difference in terms of post-operative pain control, adverse events, postoperative nausea and vomiting (PONV) and post-operative chronic breast pain.

Results: Interim results of 20 patients enrolled, comprising equal proportion of patients in each group. Age, gender, BMI and ASA scores are comparable. 4 patients in PECS group experience moderate to severe pain compared to 1 patient in the control group at 1 hour post-operatively $(\mathrm{p}=0.303)$. By 6 to 24 hours post-operatively, almost all patients in both groups had mild to no pain. Morphine usage was similar for the first 6 hours post-operatively. However, mean morphine usage for PECS group was significantly higher at 12 hours $(3.5 \mathrm{mg}$ vs $0.9 \mathrm{mg}, \mathrm{p}=0.008$ ) and 24 hours $(1.0 \mathrm{mg}$ vs $0.2 \mathrm{mg}, \mathrm{p}=0.028)$. More patients in the control group experienced intraoperative hypotension episodes (mean of 1.7 vs $0.7, p=0.087$ ) and PONV (4 vs 2 , $\mathrm{p}=0.628$ ) post-operatively. There were no PECS block related complications. Data for chronic pain is not yet available.

Conclusion: Although pain score is statistically similar in both groups, morphine usage seems to be higher in the PECS group. There is also a tendency towards lower incidence of PONV and hypotension in the PECS group. These early results suggest PECS block is comparable to IV opioids in terms of pain control and remains a promising alternative for patients with multiple co-morbidities, susceptible to opioid side effects. 


\title{
Abstract ID 57 \\ Impact of Preoperative Anxiety on Propofol Induced Hypotension in Female Patients of Elective Surgery Under General Anaesthesia: Interim Analysis From A Prospective Observational Study
}

\author{
Puneet Khanna, Lokesh Kashyap, M.K. Arora
}

Department of Anaesthesiology, Pain Medicine \& Critical Care, All India Institute of Medical Sciences, New Delhi, India

Introduction: Hypotension during induction of anaesthesia by propofol is a major concern. Patients with generalised anxiety disorder are in a state of higher baseline sympathetic activity measured by continuous minute to minute skin conductance, motor activity and $24 \mathrm{hr}$ ambient temperature. Pre-operative anxiety causes marked reduction in blood pressure after spinal anaesthesia in women undergoing caesarean delivery.

Objective: To assess the effect of preoperative anxiety on arterial pressure changes after induction with propofol.

Methods: Thirty adult (aged between $18 \& 60$ years) female patients of American Society of Anaesthesiologists' physical status I or II undergoing gynecologic surgeries have been included in this prospective observational study. Anxiety was assessed by two validated scales of anxiety namely verbal analog scale (VAS) of anxiety score ${ }^{15}$, and generalised anxiety disorder-7(GAD-7) questionnaire on the day before surgery. General anaesthesia was induced with $2 \mathrm{mg} / \mathrm{kg}$ propofol mixed with $2 \mathrm{ml}$ of $2 \%$ xylocaine over $30 \mathrm{sec}$ through an infusion pump. Systolic blood pressure, diastolic blood pressure, mean arterial pressure, heart rate was measured every $1 \mathrm{~min}$ upto $10 \mathrm{~min}$ after induction.

Results: (SD) age of the patients was 30.03(5.5) and measured median [range] of VAS anxiety and GAD-7 score were 6[2-9] and 8[0-17]. Mean (SD) of total propofol used was 141.7 (27.5) mg. A significant agreement was found between VAS and GAD-7 score ( $r^{2}=0.906, p<0.001$, Spearman's rank correlation). A statistically significant correlation was found between GAD-7 score and fall in mean arterial pressure from baseline to one minute after induction of anaesthesia $\left(r^{2}=0.380, p=0.042\right.$, Spearman's rank correlation). No correlation has been found between anxiety and change of systolic or diastolic blood pressure.

Conclusions: Our final study will recruit 100 patients; interim analysis suggests that preoperative anxiety may be a contributing factor for propofol-induced hypotension during induction of general anaesthesia in adult female patients. 


\title{
Abstract ID 58 \\ Case Report on the Retention of A Guidewire: A Rare but Avoidable Complication in Central Venous Catheterization and our Approach in Management of this Problem in a District Hospital
}

\author{
'Dhanya J, 'Ismail M, 'Saiful Aizar K, 'Muzlifah KB, 'Siti Zuraidah AK \\ 'Department of Anaesthesiology, Hospital Tuanku Ampuan Najihah, Kuala Pilah, Malaysia
}

This poster is a case report of a 38 year old Chinese gentleman with underlying heart disease that was referred to the anaesth team for shortness of breath requring Non Invasive ventilation. He was treated as Acute MCA infarct possibly secondary to cerebral emboli and infective endocarditis with underlying ischemic dilated cardiomyopathy.

During admission into ICU, we planned for long line insertion (PICC) to monitor CVP. A J-tipped guidewire was introduced through the needle after positive aspiration of venous blood. Following that the catheter was inserted. During this procedure, the guidewire was accidentally pushed further ahead into the left basilic vein and after realizing this, catheter was removed but the guidewire was missing.

Urgent X-rays of the left arm, chest and abdomen was done and noted that the guidewire was clearly visible inside the vein and migrating. Unfortunately, in view patient was unstable for transport, there was no plan to transfer. Condition worsened and we realised that time is of the essence and waiting would only complicate matters. Decision was made to proceed with the removal of guidewire in our hospital. This was done successfully via the infraclavicular approach.

No doubt guidewire retention is a much spoken about complication even in tertiery centres and the approach taken would be much more easier as assistance is always available in terms of imaging and experts to deal with this kind of situation. In a district hospital where cardiothoracic and imaging assistance is far from reach, it is important for professionals working in district hospitals to be aware of the approach that can be taken to manage this problem. 


\title{
Abstract ID 59
}

\section{Selection of the Double-lumen Tube Size for One-lung Ventilation in Asians}

\author{
IS Salwani, CHM Rahimi, ARM Ramzisham, JSM Ooi \\ ${ }^{1}$ University Kebangsaan Malaysia Medical Centre, Kuala Lumpur, Malaysia
}

Introduction: Selecting an appropriate size double-lumen tube for one-lung ventilation has always been a challenge as most choose it based on experience or using the existing guidelines based on gender and height.

Objective: This study was to determine if the appropriate choice of this tube could be based on the patients' height, weight, tracheal diameter or the left main stem bronchus diameter and also to determine the relationship between height and depth of insertion amongst Asians.

Methods: This was a retrospective review of 179 patients who used a left-sided double-lumen tube and also had a posterior-anterior view of a digital chest radiograph for tracheal and left main bronchus diameter measurements. Additional data collected included patients' demographics and double-lumen tube size used.

Results: There were $123(68.7 \%)$ males and $56(31.3 \%)$ females with an overall mean age of $33.3 \pm 16.3$ years. Majority of the males $(48.8 \%$ ) used a size $39 \mathrm{Fr}$ while and females $(46.4 \%)$ used a $35 \mathrm{Fr}$. There were significant but weak correlations between double-lumen tube size with height (male: $p<0.001, R^{2}=0.222$; female: $p=0.001, R^{2}=0.193$ ) and weight (male: $p<0.001, R^{2}=0.109$; female: $p<0.001, R^{2}=0.211$ ) and no significant correlation between the tube size with tracheal and left main bronchus diameter. Depth of double-lumen tube inserted was significantly correlated to patient's height.

Conclusions: The appropriate size of the double-lumen tube could not be predicted based on patients' height, weight, tracheal or left main bronchus diameter alone in Asians but depth of insertion of the tube was dependent on the height in both genders. 


\title{
Abstract ID 60
}

\section{An Audit Of Epidural Analgesia (EA) Among Patients Undergoing Abdominal Surgery in Hospital Umum Sarawak (HUS)}

\author{
KY Loo', YY Tan', CP Jap', PN Ng ${ }^{2}$, MY Tang ${ }^{3}$ \\ ${ }^{1}$ Hospital Umum Sarawak, Kuching, Malaysia \\ ${ }^{2}$ Hospital Kuala Lumpur, Kuala Lumpur, Malaysia \\ ${ }^{3}$ Faculty of Medicine and Health Sciences, Universiti Malaysia Sarawak, Kota Samarahan, Malaysia
}

Objectives: To look at the practice and outcome of EA in abdominal surgeries in HUS, and to identify causes of suboptimal EA.

Methods: This is a prospective study on all adult patients undergoing open abdominal surgery (General, Hepatobiliary, Urosurgery, Gynaecology, Vascular and Plastic surgeries) from February to May, 2016. Patient's data, suitability for EA or reasons of unsuitability, epidural catheterization and its details were recorded intraoperatively. The outcome data on postoperative pain control and complication were obtained from Acute Pain Service database.

Results: 377 adult patients had open abdominal surgery within the study period. 167 (44.3\%) patients were suitable for EA. Common reasons for unsuitability were small surgical incision (22\%), preexisting or anticipated coagulopathy (14.1\%) and local or systemic infection (9.8\%). Out of the 167 suitable patients, only $88(52.7 \%)$ patients had epidural catheter inserted intraoperatively. Reasons of epidural catheter not inserted in the other patients were insufficient OT time (18.6\%), patient's refusal (16.8\%), and failed insertion attempt (6.0\%). There were no significant identifiable patient factors (age, gender, weight, ASA class) or surgical factors (types of surgery and urgency of surgery) which predicts if a patient suitable for EA will receive EA eventually. Out of the 88 patients who had EA, only 56 (63.64\%) patients achieved adequate analgesia. Contributing factors to inadequate analgesia were inadequate epidural infusion (53.1\%), catheter dislodged or displaced (28.1\%) and failed epidural blockade (18.7\%). As many as $23(26.1 \%)$ epidural catheter was inserted at inappropriate spinal level. There was no major complication from EA.

Conclusions: EA only contributed to approximately $21 \%$ of perioperative analgesia for abdominal surgeries at HUS. Insufficient OT time and failed epidural catheter insertion were modifiable factors contributing low EA rate. Inadequate epidural infusion, catheter dislodged or displaced, insertion at inappropriate spinal level contributed to suboptimal EA. 


\title{
Abstract ID 61
}

\section{Assessment Of The Depth Of Endotracheal Tube In Pediatric Using Fluoroscopy Technique: A Pilot Study.}

\author{
Hasmizy M'1, W Lynn Xuan², Siti Baiduri B', Hanafi S', Norzalina E'. \\ ${ }^{1}$ Department of Cardiothoracic Anaesthesiology and Perfusion, Sarawak Heart Centre, Kota Samarahan, \\ Sarawak. \\ ${ }^{2}$ Department of Anesthesiology and Intensive Care, Sarawak General Hospital, Kuching, Sarawak
}

Background: In children, precise depth of tracheal tube insertion is important to avoid inadvertent tracheal extubation or endobronchial intubation. We practiced endotracheal tube length in centimeter at the patient lip based on formula, age/2 +12 for children more than 1 year old and endotracheal tube internal diameter $\times 3$ for children less than 1 year old.

Objective: The aim of this study was to determine the accuracy of standard practice for estimating oral tracheal tube length in children by using fluoroscopy.

Methods: We performed orotracheal intubation in pediatric patients undergoing cardiac catheterization. ETT length was calculated based on age formula (ET1). However, the final ETT length placement will depends on equal auscultation of bilateral breath sounds (ET2). Fluoroscopy was used to measure the distance between the carina and the distal tip of the ETT $(D)$. Actual distance calculated ETT $\left(D_{A}\right)$ between the carina and the distal tip of the ETT was D - (ET1- ET2).

Results: Fourty- two children aged between 0.11 and 10 years (median $4.7 \mathrm{yr}$ ) were studied. In ET2, no endobronchial intubation, 3 patients $(7.1 \%)$ had ETT $<0.5 \mathrm{~cm}$ from the carina and 36 patients $(85.7 \%)$ had $\mathrm{ETT}>1 \mathrm{~cm}$ from the carina. In $\mathrm{D}_{\mathrm{A}}, 5$ patients $(11.9 \%)$ will have endobronchial intubation while 7 patients $(16.7 \%)$ will have ETT $<0.5 \mathrm{~cm}$ from the carina and 22 patients $(52.3 \%)$ will have ETT above $1 \mathrm{~cm}$ from the carina.

Conclusion: This study showed that by using ETT length age-formula alone without clinical assessment will results in high incidence of endobronchial intubation and low placement of the ETT. 


\title{
Abstract ID 66
}

\section{Delivering Safe Anaesthesia For Electroconvulsive Therapy During Pregnancy}

\author{
I Kong, K Tan, P Tan \\ Sarawak General Hospital, Kuching, Sarawak
}

Background: Treatment of psychiatric illnesses poses challenges during pregnancy. Many psychotropic medications have teratogenic effects. Furthermore, medication-resistant life threatening mental deterioration may require rapid and effective interventions. Electroconvulsive therapy (ECT) offers speed of clinical recovery. It is a relatively safe and effective treatment during pregnancy but its anaesthetic management involves particular considerations.

Case Report: We illustrate a case of 29 years old, $50 \mathrm{~kg}$ lady pregnant with her third child at 24 weeks. She has underlying schizophrenia but defaulted treatment. She was brought in to Emergency Department with history of verbally abusive speech, grandiose delusions, insomnia and self-abuse for a few months. She was started on oral haloperidol up to $3 \mathrm{mg}$ twice daily for three days followed by oral olanzapine $5 \mathrm{mg}$ and promethazine $50 \mathrm{mg}$. However, she continued to be aggressive and delusional requiring frequent intramuscular injections of haloperidol. ECT was planned by the psychiatric team. She was kept fasted for six hours, given anti-aspiration prophylaxis and intubated after rapid sequence induction (RSI) with intravenous fentanyl $100 \mathrm{mcg}$, propofol $60 \mathrm{mg}$ and suxamethonium $50 \mathrm{mg}$. Pre- and post-ECT foetal scans were done by the obstetric team during the first two sessions. Subsequently, foetal monitoring was performed by Doppler. A total of six ECT were delivered over two weeks and the patient improved markedly.

Learning points: It is important to understand the physiologic responses to electrical stimulus in order to optimise the anaesthetic management. Moreover, delivering a safe anaesthetic to an obstetric psychotic patient with risk of aspiration and potential difficult airway in a remote location is challenging. Other related concerns are the choice and dosage of drugs to achieve a balance between providing an adequate anaesthesia (RSI) for intubation and ECT without adversely affecting the efficacy of ECT, as well as monitoring foetus for premature labour and spontaneous abortion post-procedurally. 


\title{
Abstract ID 67
}

\section{Underdosing Of Intraoperative Opioids, A Glaring Shortcoming In Perioperative Pain Management}

\author{
Ummu YH Hamid', Lily Hii ${ }^{1}$, Mee Yee Tang ${ }^{2}$ \\ ${ }^{1}$ Hospital Umum Sarawak, Kuching, Malaysia \\ 2 Universiti Malaysia Sarawak, Kota Samarahan, Malaysia
}

Background: While multimodal analgesia is advocated for perioperative pain management, opioids remain the mainstay of perioperative analgesia. The under-dosing of intraoperative opioids among anaesthetists may have contributed to poor perioperative pain management.

Objectives: To compare the anaesthetist's opioid dosing intraoperatively to the patient's opioid usage postoperatively and to identify factors that affect anaesthetist's opioid dosing intraoperatively.

Methods: This is a pilot observational study of four weeks duration. All patients who were prescribed patient-controlled analgesia with Morphine (PCAM) for postoperative analgesia were included. The conduct of anaesthesia followed the individual anaesthetist's discretion. Patient's age, gender and weight, site and duration of surgery, intraoperative Morphine doses given by anaesthetist, postoperative Morphine doses used via PCAM in the first six hours and the next eighteen hours were recorded. Statistical analyses on the ratio of postoperative to intraoperative opioid dosing, the factors affecting intraoperative opioid dosing were done.

Results: 61 patients were included, 31 men and 30 women. Their age (years), weight (kilogram) and duration of surgery (hours) in mean \pm standard deviation were $42.1 \pm 16.7,61.8 \pm 15.0$ and $3.6 \pm 3.1$ respectively. Patient's age, gender, weight, the site and duration of surgery do not significantly affect the anaesthetist's Morphine dosing intraoperatively. After controlled for the duration of surgery, the ratio of postoperative to intraoperative Morphine dosing in median (interquartile range) at the first six hours and the next eighteen hours for abdominal surgery were $0.41(0.18,0.9)$ and $019(0.04,0.33)$; for peripheral surgery $0.84(0.22$, $2.21)$ and $0.18(0.03,0.41)$; and for spine surgery $0.44(0.2,1.91)$ and $0.25(0.08,0.57)$.

Conclusions: Patient's Morphine usage at the first six hours postoperatively was similar if not higher compared to the anaesthetist's opioid dosing intraoperatively especially for peripheral surgery and spine surgery. Anaesthetist's opioid dosing intraoperatively is not associated by patient's age, gender, weight, the site and duration of surgery. 


\title{
Abstract ID 68
}

\section{Implementing Rapid Emergence And Early Extubation From General Anaesthesia}

\author{
Ruwaida Isa, Jamaliah Said, Suki b. Ismet, Nor Hidayah Zainool Abidin, Norhashim b \\ Sulaiman, Ahmad Azim Mohamed. \\ ${ }^{1}$ Hospital Raja Perempuan Zainab 2, Kota Bharu, Kelantan.
}

Background: Quality of improvement in the field of anaesthesiology has focus on intubation rather than extubation process. Proper and faster removal of ETT after general anaesthesia requires skill and judgement learned through experience. Complications during emergence and extubation from general anaesthesia is not uncommon, it carries significant effect to the patient, anaesthesia providers, surgeon. This study will highlight that with proper flow and planned emergence technique, the process of awakening and extubation will be fast and more efficient.

Objectives: To develop a faster process of emergence from general anesthesia and to reduce unnecessary delay due to prolonged emergence, and complications during extubation process. To identify the reason of slow in emergence and extubation from general anesthesia. To encounter the problems and other factors that contributes to slow emergence and extubation process in General Anesthesia. To plan remedial actions to reduce this incidence of slow extubation after general anaesthesia and then to re-evaluate the incidence after remedial action taken.

Methods: A retrospective verification study was conducted in HRPZ II in December 2014. It was found that as the percentage of fast emergence and extubation of patient after general anaesthesia was as low 52 $\%$. It was also found that $43.5 \%$ of anaesthesia doctors have experience of less than 2 years. Subsequently a cross-sectional study was done. Questionnaire was given to anaesthetics medical officers and specialist in assessing their knowledge and practice regarding these matters. Remedial measures were performed in April till June. 2015.

Results: Our results from questionnaire conducted, most of anaesthesia doctors in HRPZ 2 preferred sevoflurane and isoflurane as maintainance agent in general anaesthesia (99\%). $73 \%$ of anaesthesia doctors prefer to promote spontaneous breathing/off IPPV after completion of surgery. $91.3 \%$ of anaesthesia doctors prefers to give reversal after completion of surgery. This was associated with slow extubation process where our observational study found that $47.8 \%(\mathrm{~N}=23)$ has fast extubation after general anaesthesia while $47.9 \%$ $(\mathrm{N}=23)$ takes longer extubation time. Remedial measure were focused on a planned emergence technique where anaesthetist prepare to switch technique and inhalational agent during operation together with timing medication and agent according to estimated time before end of surgery. We also focused on having standard extubation checklist that will inculcate discipline of higher performance and promote minimum necessary standard for a fast yet safer extubation process.

Conclusion: Remedial measures done during the study has successfully instilled practice of fast emergence and extubation techniques together with a better documentations regarding patient extubation process. It is hope that this study will also become a stepping-stone for further studies to improve recovery from anaesthesia after elective surgery. 


\title{
Abstract ID 70
}

\section{A Crossover Study Comparing In-Plane and Out-of-Plane Techniques for Simulated Ultrasound-Guided Central Venous Cannulation on Phantom Models by Trainees in Anaesthesiology}

\author{
Yap Ka Boi ${ }^{1}$, Lee Yan Wei', Kamal-Bashar Abu Bakar', Liu Chian Yong'2, Joanna Ooi Su \\ Min $^{2}$, Muhammad Maaya ${ }^{2}$ \\ ${ }^{1}$ Hospital Kuala Lumpur, Kuala Lumpur, Malaysia \\ ${ }^{2}$ Universiti Kebangsaan Malaysia Medical Centre, Kuala Lumpur, Malaysia
}

Background: In-plane and out-of-plane, the two common approaches to ultrasound-guided venous cannulation, can result in posterior venous wall puncture especially in novices.

Objectives: This crossover study compared the incidence of posterior vessel wall puncture between these two approaches during ultrasound-guided simulated central venous cannulation by anaesthesiology trainees.

Methods: Each phantom model, simulating a central vein and artery, was cannulated by 37 anaesthesiology trainees under ultrasound-guidance using the in-plane approach (IPA) and out-of-plane approach (OPA). Total procedural time, which included the time taken from starting image scanning until commencing puncture, was recorded. The number of attempts required to achieve successful venous cannulation was noted. Finally, the models were examined for posterior venous wall and arterial puncture.

Results: Total procedural time was shorter with the OPA ( $26.5 \mathrm{vs} 50.3 \mathrm{sec}, p=0.001)$. The time taken from starting image scanning until commencing puncture was quicker for the OPA ( $2.2 \mathrm{vs} 12.3 \mathrm{sec}, p<0.0001)$. The IPA resulted in significantly more attempts for cannulation. Twenty and eleven participants were successful within the first pass using the OPA and IPA, respectively $(p=0.034)$. There was no statistical difference in the incidence of posterior vessel wall puncture between these two techniques. The OPA had significantly less arterial puncture compared to the IPA ( 2 vs $9, p=0.022$ ).

Conclusion: There was a comparable incidence of posterior vessel wall puncture between the in-plane and out-of-plane techniques during ultrasound-guided simulated central venous cannulation by anaesthesiology trainees. 


\section{Abstract ID 71 \\ Postoperative Recovery Profiles After Ambulatory Anaesthesia Using Spectral Entropy}

Md Nor, Nadia. MBBCh BAO (Ireland), MMed (UKM), Zainuddin, Muhammad Zurrusydi. MBBS (IIUM), Dr Anaesth\&CritCare (UKM), Wan Mat, Wan Rahiza. MB ChB (Otago), MMed (UKM), Masdar, Azlina. MD (UKM), FCAl, Associate Prof Dr Raha Abdul Rahman MB BCh Bao(NUI), MMed (UKM).

All are affiliated Department of Anaesthesiology and Intensive Care, Faculty of Medicine, Universiti Kebangsaan Malaysia Medical Centre, Kuala Lumpur, Malaysia.

Background: Anaesthesia for ambulatory surgery affects postoperative patient recovery impacting on safe discharge home.

Objective: To compare postoperative recovery profiles between patients with spectral entropy and patients without spectral entropy monitoring, after sevoflurane anaesthesia, in the day care setting.

Methods: This prospective, randomised, double-blind controlled study compared postoperative patient recovery profiles after sevoflurane anaesthesia, with and without spectral entropy monitoring. Forty ASA I-II patients aged between 18 to 65 years, scheduled for ambulatory surgery were recruited. They were randomised into Group A (without spectral entropy monitoring) and Group B (with spectral entropy monitoring). In Group A, sevoflurane was titrated to prevent awareness while maintaining haemodynamic parameters within $20 \%$ of baseline values. In group B, sevoflurane was titrated to maintain state entropy (SE) and response entropy (RE) values within the range of 40-60.

Results: Patient demographic, duration of anaesthesia and surgery, and accumulative intraoperative propofol and fentanyl were comparable between the groups. Mean end-tidal concentration of sevoflurane and total amount of sevoflurane used was significantly lower in Group B. This corresponded with significantly higher $\mathrm{SE}$ and RE values with spectral entropy monitoring, $\mathrm{p}<0.05$. Postoperative patient recovery profiles were also significantly better in Group B, $p<0.05$. Significantly more patients in group B achieved a minimal modified Aldrete score of 10 on arrival in the phase I recovery area, and at three hours scored higher mean quality of recovery score, $\mathrm{p}<0.05$.

Conclusion: Spectral entropy monitoring improved postoperative recovery profiles after ambulatory surgery using sevoflurane without compromising on patient safety or imposing additional side effects to the patient. 


\title{
Abstract ID 73
}

\section{A Comparison Of Depth Of Muscle Relaxation To Improve Performance Of Thermal Ablation For Liver Tumours}

\author{
S.Masohood N', PS Loh' \\ ${ }^{1}$ University of Malaya, Kuala Lumpur, Malaysia
}

Percutaneous thermal ablation of hepatic tumours is one of the main treatment modality for small primary hepatocellular carcinoma (HCC) and liver metastases. Movement by patients during these ablation procedures may reduce the accuracy of targeting the tumour and increase the risk of complications. Large hepatic motion during breathing would also cause technical difficulties for the interventional radiologist to perform the procedure safely.

Recent studies have shown that deep neuromuscular blockade improves surgical conditions in laparoscopic surgery by improving visibility in the surgical field and reducing involuntary movements. This study hopes to prove that deep neuromuscular block will improve the performance of microwave ablation by radiologist. The main objective of this study is to compare the different depths of neuromuscular block between deep neuromuscular blockade (DMB) and moderate neuromuscular blockade (MMB) groups during microwave ablation (MWA) for primary or secondary hepatic tumours to look at the ease of performing the MWA by the interventional radiologist.

This was a single-centre, prospective, double-blinded, randomized controlled trial involving 50 patients undergoing computed tomography (CT) guided microwave ablation (MWA) of primary and secondary liver tumours in Department of Biomedical Imaging, University Malaya Medical Centre from January 2015 to November 2015. This study is registered with National Medical Research Register (NMRR ID 15-51-24027) and the University Malaya Medical Centre Ethics Committee (MECID No. 20151-930) approved the study protocol.

The mean performance score by radiologist in the DMB group was higher (mean 4.5, SD \pm 0.59 ) compared to the MMB group (mean 3.6, $S D \pm 0.85)(p<0.01)$. There is a mean difference in the performance score of 0.9 between the two groups. This study proved that deep neuromuscular blockade improves performance of microwave ablation of hepatic malignancy. However, it did not reduce the complications of MWA nor did it shorten procedure duration. 


\title{
Abstract ID 74
}

\section{Awake Craniotomy: Are They Satisfied?}

\author{
Fadhli Suhaimi Abdul Sukur', V.Sivanaser, S.Velayuthapillai' \\ ${ }^{1}$ Hospital Kuala Lumpur, Kuala Lumpur, Malaysia
}

Background: Awake craniotomy is a well-established neurosurgical procedure. Its surgical benefits are well documented. It allows for the avoidance of general anesthetic effects on the intraoperative neurological monitoring while reducing ICU stay. A total of 27 patients underwent awake neurosurgical procedures since 2014 at HKL, 14 patient underwent awake craniotomy under conscious sedation while 12 underwent Burr Hole under local anaesthesia. There is lack of data regarding patient satisfaction following procedure.

Objective: To assess patient's satisfaction following an awake craniotomy under conscious sedation.

Method: A prospective qualitative study was undertaken on patients undergoing awake craniotomy for tumor surgery using an adapted questionnaire from the Royal College of Surgeon. The questionnaire is divided into pre-operative, intra and post-operative parts .A respond to each question is required for completion of the questionnaire. Doctors who did not partake in the initial procedure conducted the interview via a phone call.

Result: Out of the 14 patients recruited, 10 patients completed the questionnaire, 2 patients died and 2 patients were uncontactable. $80 \%$ of patients understood the anaesthesia explanation and information given during preoperative visit. The remaining $20 \%$ felt that excessive information was given. All patients were able to recall important events such as clamping of the scalp with Mayfield clamps, positioning and intraoperative mapping during the intraoperative period. $80 \%$ of them felt comfortable throughout procedure Main reasons cited for discomfort during procedure in $20 \%$ of patients were surgical pain, cold, full distension and position. Two patients experience pain scores of more than 4 (mean of 6 ). $100 \%$ of patients were pain free with no nausea or vomiting at the recovery period.

Conclusion: Awake craniotomy with conscious sedation is well tolerate by patients at our institute. Methods to control intraoperative pain and discomfort needs to be addressed. 


\title{
Abstract ID 75
}

\section{Epidural Analgesia for Labour: Comparison between Pre-puncture Ultrasound Localisation versus Palpation Technique}

\author{
Nahemah Hasanaly', Thohiroh Abdul Razak', Muhammad Maaya², Joanna Ooi Su Min², \\ Liu Chian Yong ${ }^{2}$ \\ ${ }^{1}$ Hospital Kuala Lumpur, Kuala Lumpur, Malaysia \\ 2 Universiti Kebangsaan Malaysia Medical Centre, Kuala Lumpur, Malaysia
}

Introduction: Epidural analgesia is the preferred method of pain relieve during labour. Performing an epidural block in a parturient in labour is always challenging especially in locating the intervertebral space, hence routine use of ultrasound has been recommended.

Objectives: This prospective, randomized study compared the number of attempts, time taken and the desired intervertebral space for epidural catheter insertion by using pre-puncture ultrasound localization or palpation technique.

Methodology: Sixty six ASA I/II parturient in early phase of labour were randomized into two groups: Group U (pre-puncture ultrasound, $n=33$ ) or Group P (palpation technique, $n=33$ ) for localization of the intervertebral space for Tuohy needle insertion in labour epidural.

Results: Patients from Group U had a significant higher first successful attempt than Group P $(60.6 \%$ vs $33.3 \%, p=0.042)$. The median number of attempts was lesser in Group U compared to Group P [1 (1-2) vs $2(1-3), p=0.011]$. All parturient from Group $U$ had correct intervertebral space for insertion of Tuohy needle compared to Group P ( $100 \%$ vs $84.8 \%, p=0.027)$. However, the mean time taken for localization of the intervertebral space was significantly longer in Group U ( $341.8 \pm 65.4 \mathrm{sec}$ vs $15.8 \pm 7.2 \mathrm{sec}, \mathrm{p}<0.001)$. There were no complications recorded.

Conclusion: Pre-puncture ultrasound localization of intervertebral space for Tuohy needle insertion in labour epidural was more accurate, achieving a higher success rate with fewer attempts compared to the palpation method. 


\title{
Abstract ID 77
}

\section{Clinical Audit on 'Decision-to-Delivery Interval' in Caesarean Sections at Maternity Unit, Hospital Pulau Pinang.}

\author{
ML Lee, S Cheah, A Lee, KY Boon, XF Lim, J Ong, SS Lim, SH Lim, J Hassan \\ Department of Anaesthesiology and Intensive Care, Hospital Pulau Pinang, Malaysia
}

Introduction The rate of caesarean deliveries is increasing steadily over the years. The National Institute of Clinical Excellence (NICE) in UK recommended a 4-point system to classify the urgency of caesarean sections (CS) to better manage resources and prioritise urgent CS over elective cases. In Category 1 CS where foetal or maternal compromise exists, a multidisciplinary teamwork approach is vital to ensure a safe and expedited delivery. The decision-to-delivery interval (DDI) defines the timeline between a decision for caesarean and the delivery of the baby. The NICE guidelines for CS in 2011 suggested a 30-min DDI for Category $1 \mathrm{CS}$ and 75 -min DDI for Category $2 \mathrm{CS}$, as an audit standard to measure the overall performance of a maternity unit.

Objectives. We aim to audit and classify all CS performed by the Maternity Unit Hospital Pulau Pinang (HPP). The DDI derived will be compared with international standards. Potential causes for delay in DDI will be identified if any.

Methods A prospective cohort study for all CS conducted by the Maternity Unit HPP from March $1^{\text {st }}$ to May $31^{\text {st }}$ 2016. DDI for all CS performed will be derived and proportions of DDI that complies with recommended standards are calculated. A general linear model analysis will be performed to identify factors influencing the DDI.

Findings. The DDI exceeded recommended interval in $>70 \%$ of Category-1 CS, but only $19 \%$ in Category-2 CS. Main factor delaying DDI is the location of the CS outside the Maternity Unit building.

Conclusion. The audits highlights the low compliance with target DDI in urgent Category-1 CS. Education to increase awareness on different levels of urgency in CS and specific protocols to enhance efficiency and shorten pathways to surgery should be considered. 


\title{
Abstract ID 78
}

\section{The ABCs Of Managing A Patient With Marquio Syndrome}

\author{
Noorjahan Haneem Md Hashim, Lucy Chan \\ ${ }^{1}$ University of Malaya, Kuala Lumpur, Malaysia
}

Introduction: Marquio syndrome is an autosomal recessive condition where patients are born with $\mathrm{N}$-acetylgalactosamine-6 sulfatase deficiency. This condition causes multiple deposits of glycosaminoglycans on tissues and organs causing multiple problems. Though the complications from these deposits can be serious, they affect these patients differently.

Report: We report our management of a 29 year old lady, weighing $19 \mathrm{~kg}$ and height of $91 \mathrm{~cm}$. She was referred to our institution for elective bilateral femoral osteotomy for correction of bilateral genu valrum. Pre-anaesthesia assessment revealed these possible threats to life to arise perioperatively:

Difficult airway, with history of failed fibreoptic intubation in a tertiary centre, multiple airway structural abnormalities and severe limitation in cervical movement

Risk of perioperative hypoxaemia, from severe restriction of respiratory function from ribs abnormality and kyphoscoliosis

Difficult intravenous access, from joint deformity and limitation in movement

Patient was counseled multiple times regarding her care. She was also referred to a paediatric ENT consultant of airway assessment pre-operatively.

Anaesthesia care was tailored to support her physiological needs during the surgery and reduce her perioperative risks by managing her $\mathrm{ABCs}$.

Conclusion: It is important to recognize the potential threats to life when managing patients with Marquio syndrome. As each patient born with this syndrome presents differently, early pre-anaesthesia consult, involving patients in decision making, multidisciplinary input, pre-anaesthesia optimization and preparation, aggressive physiotherapy and optimal analgesia ensures these patients' anaesthesia experience are safe and comfortable. 


\title{
Abstract ID 79
}

\section{The Spreading Of Labor Analgesic Concerns By A Marketing Theory In A Private Japanese Hospital}

\author{
Shingo Irikoma, Yoshie Toba, Sotaro Kokubo \\ Department of Anesthesiology, Seirei Hamamatsu General Hospital, Hamamatsu, Japan
}

Objectives: Labor analgesia is gradually spreading among Japanese pregnant women in recent years. Because an intensification of delivery is difficult in Japan, obstetric anesthesiologists cannot work in only labor ward. Though the need for labor analgesia is increasing, there are not many institutes can provide labor analgesia in Japan. So we have trained obstetricians to be able to insert the epidural catheterization and manage the labor analgesia. Midwives also support the labor analgesia management. Cases of labor analgesia have increased in our hospital in recent 3 years. We retrospectively researched our new labor analgesia system from the point of view of marketing theory.

Methods: This study was approved by the Institutional Review Board in our hospital. We investigated new our labor analgesia project and system from the point of view of marketing theory July in 2013 to June in 2016. We tried to explain the factor of our successful project to use the $5 \mathrm{C}$ analysis and the marketing $\operatorname{mix}(4 \mathrm{P})$.

Results: There are the $5 \mathrm{C}$ analysis and the marketing mix (4P) in the marketing theory. The $5 \mathrm{C}$ are company, consumer, customer competitor and community. The marketing $4 \mathrm{P}$ are product, price, place and promotion. The key factors of our successful labor analgesia project and novel were management resource about general hospital (company in $5 \mathrm{C}$ ) and low frequency of breakthrough pain because of quick responses by obstetricians and midwives (product in 4P). Monthly average number of cases with labor analgesia was 8 , $15,23,30$ cases in 2013, 2014, 2015, 2016, respectively.

Conclusions: We unwittingly used the marketing theory and increased number of cases with labor analgesia in our private hospital in Japan. 


\title{
Abstract ID 80
}

\section{Turner's With Diabetes Inspidus: A Case Of Severe Underweight With High Water Loss}

\author{
Dr Nadia Aqilla Jawardi, Dr Fadhli² \\ ${ }^{1}$ Hospital Kuala Lumpur, Kuala Lumpur, Malaysia
}

We report a case of a 17 years old girl with underlying Mosaic Turner syndrome diagnosed with pituitary stalk germinoma since 2014. She is taking premalin and L-thyroxine because of hormonal deficiency. Her BMI is only 15.2 ( height $128 \mathrm{~cm}$, wight $25 \mathrm{~kg}$ ). She underwent right supraorbital craniotomy because repeated $M R I$ showed increment in tumor size. Induction and intubation were uneventful and anaesthesia maintained with total intravenous anaesthesia. Arterial blood gas (ABG) baseline within normal range with sodium $135 \mathrm{mmol} / \mathrm{L}$.

Intraoperatively, after 1.5 hours, patient developed sudden tachycardia up to 130 beats per minute. She was still normotensive and afebrile. Condition remains the same despite increasing remifentanil an propofol infusion. Urine output pouring out up to $450 \mathrm{cc}$ within an hour, and for the subsequent hours, urine output was $200 \mathrm{cc} /$ hour $(8 \mathrm{cc} / \mathrm{kg} /$ hour). Serial ABG repeated hourly and revealed worsening metabolic acidosis with hypernatremia (sodium 155mmol/L). iv Desmopressin $1 \mathrm{mcg}$ given in view of possible diabetes insipidus, and for the following hours, urine output improving to $100 \mathrm{cc} /$ hour.

Patient was kept intubated post operatively, and successfully extubated on the following morning. Urine osmolality was $89 \mathrm{mOsm} / \mathrm{kg}$ and serum osmolality was $306 \mathrm{mOsm} / \mathrm{kg}$. Serum sodium ranging from 133 to $145 \mathrm{mmol} / \mathrm{L}$. She still requiring s/c Desmopressin daily dose up to 4days post operatively because of polyuria. We discussed a case of Turner's syndrome patient with BMI of 15.2, developed sudden intraoperative diabetes insipidus that can leads to rapid deteroriation due to dehydration, acidosis and hypernatremia. Early diagnosis, correction of fluid loss and prompt treatment and management is mandatory 


\title{
Abstract ID 81
}

\section{Total Intravenous Anaesthesia For Caesarean Delivery: Experience In Hospital Sultanah Nur Zahirah, Kuala Terengganu}

\author{
N Razak \\ ${ }^{1}$ Department of Anaesthesia \& Intensive Care, Hospital Sultanah Nur Zahirah, K Terengganu, Malaysia.
}

Introduction: These case series illustrate our experienced on total intravenous anaesthesia (TIVA) for cesarean delivery. Following informed consent, five patients were planned for TIVA during cesarean delivery. Four patients were having symptomatic intracranial pathology and one patient was ventilated with high ventilator setting in intensive care unit; who were planned for cesarean delivery.

Report: All patients were induced with $1 \mathrm{mcg} / \mathrm{kg}$ slow bolus of intravenous Remifentanil, followed by intravenous Propofol $2.5 \mathrm{mg} / \mathrm{kg}$. Once patient had loss of consciousness, cricoid pressure was applied and intravenous suxamethonium $1 \mathrm{mg} / \mathrm{kg}$ was administered. Airway was maintained with endotracheal tube. Patient was monitored with standard monitoring and BIS monitor. The anaesthesia was maintained with $\mathrm{TCl}$ Propofol at a plasma concentration of $4-5 \mathrm{mcg} / \mathrm{ml}$ and infusion Remifentanil at 0.1-0.2 $\mathrm{mcg} / \mathrm{kg} / \mathrm{min}$ before delivery of the baby. Hemodynamically was maintained stable throughout the cesarean section. Only one baby required intubation due to severe prematurity. Four babies delivered with good Apgar Score. None of the babies required naloxone.

\section{Conclusion:}

TIVA is not for routine anaesthesia for cesarean section. TIVA can be an alternative of anesthesia in some circumstances, e.g., when there is coexisting maternal disease, increases in maternal heart rate and blood pressure may be detrimental. TIVA can be used provided adequate paediatric support is present to manage brief respiratory depression in the neonate. 


\title{
Abstract ID 82
}

Case Report: Successful Resuscitative Hysterotomy in A Parturient with Cardiopulmonary Arrest in the Antenatal Patient Admission Center (PAC)

\author{
ML Lee, J Ong, CH Lim, J Hassan \\ ${ }^{1}$ Department of Anaesthesia \& Intensive Care, Hospital Pulau Pinang, Malaysia.
}

Background: Cardiopulmonary arrest in pregnancy is a challenging situation that requires quick decisions and prompt interventions to maximize the chance of a successful resuscitation. Resuscitative hysterotomy $(\mathrm{RH})$ can potentially provide substantial benefit to both the mother and baby and should be considered for all cardiac arrest parturients with uterine fundus palpable at or above the umbilicus. The concept of $\mathrm{RH}$ focuses on the physiological benefit of abolishing the effect of aortacaval compression caused by the gravid uterus and improving the chance of return of spontaneous circulation (ROSC) during resuscitation of the collapsed parturient. RH and delivery should be performed regardless of the foetal viability and the duration of the arrest and this departs from the older term 'perimortem caesarean delivery' which has more fetocentric indications.

Case: We report a case of a 34 year old parturient with 2 previous scars who presented to the antenatal PAC with abdominal pain and severe metabolic acidosis. She subsequently arrested and was not responsive to cardiopulmonary resuscitations (CPR) for almost $30 \mathrm{~min}$. Onsite RH was performed and there was immediate ROSC upon delivery of the fetus. She had a prolonged stay in the ICU complicated with sepsis and venous thromboembolism (VTE) but was able to make a successful recovery with full neurological function.

Conclusion: Good organization of response to cardiopulmonary arrest in pregnancy is critical to the success of any resuscitation. Written guidelines, regular simulations, skills training and a robust response to crisis system needs to be in place in order to achieve the best possible outcome in both the mother and the baby. 